\section{Variability in Reaction to Root and Crown Rot Caused by Rhizoctonia Solani Among Table Beet Cultivars, Breeding Lines, and Plant Introductions in Controlled Environment Conditions}

\author{
Katharina S. Wigg and Irwin L. Goldman \\ Department of Horticulture, University of Wisconsin-Madison, 1575 Linden \\ Drive, Madison, WI 53706
}

Additional index words. Beta vulgaris ssp. vulgaris, pocket rot, Rhizoctonia root and crown rot, host plant resistance

\begin{abstract}
Table beet (Beta vulgaris ssp. vulgaris) is adversely affected by the soilborne fungus, Rhizoctonia solani, which greatly decreases root quality. There are no reports of breeding efforts designed to improve resistance to this fungus in table beet. A collection of table beet PIs, cultivars, and publicly available inbreds were characterized for their response to inoculation with $R$. solani in three replicated greenhouse experiments conducted between 2017 and 2019. An isolate of AG 2-2 IIIB was used to inoculate 8week old plants, and both susceptible and resistant sugar beet genotypes were used for comparison. Plants were evaluated for internal and external disease characteristics 3 and 5 weeks postinoculation. Compared with uninoculated controls, PI accessions ranged from $2 \%$ to $44 \%$ infection, whereas cultivars and inbred lines ranged from $16 \%$ to $53 \%$ and $22 \%$ to $52 \%$, respectively. No commercial cultivar outperformed the resistant sugar beet genotype; however, several PI accessions exhibited lower mean diseased tissue percentages than the resistant sugar beet. This suggests that resistance to $R$. solani may exist in table beet germplasm. PI accessions exhibited greater resistance on average than did cultivars. Inbred lines exhibited the least amount of variation. Ordinal regression allowed for prediction of infection response, which may be helpful in future selection efforts. 'Badger Flame', Ames 22164, PI 502294, PI 169015, 'Long Season', 'Red Cloud', and 'Albina Vereduna' were some of the most resistant accessions in our screens. The findings from this study can be used to inform decisions for breeding for host plant resistance in table beet.
\end{abstract}

Table beet (Beta vulgaris L. ssp. vulgaris) is a regionally important crop in the United States. Wisconsin leads U.S. production with more than 1600 ha devoted to production (U.S. Department of Agriculture, 2017). A significant detriment to table beet quality and yield is Rhizoctonia root and crown rot caused by the fungus Rhizoctonia solani Kühn (Abawi et al., 1986; Natti, 1953; Pethybridge et al., 2018), which renders table beet roots unmarketable. Currently, there are few chemical options for controlling this fungus in table beet production, and no reports of efforts to improve host plant resistance through breeding.

$R$. solani is a soilborne necrotrophic pathogen affecting many crop families. Within the Rhizoctonia complex, 12 anastomosis groups (AGs) have been described. These AGs distinguish genetically diverse isolates according to their affinity for hyphal fusion with one another (Ogoshi, 1985). Several AGs have been further divided into "intraspecific groups" (ISGs) (Ogoshi, 1987), or more appropriately "subgroups" (SGs) (Ogoshi, 1996) to categorize isolates by their biochemical, pathogenic, and genetic quali2-2 causing root rot and AGs 1, 2-2 (Naito et al., 1978), and 4 infecting the crown and foliage (Naito et al., 1976). A study by Yamaguchi et al. (1977) confirmed that isolates of AG 2-2 caused severe disease on sugar beet roots compared with those of AG 1 and 4. AGs 2-2-IIIB (Büttner et al., 2002; Strausbaugh et al., 2011; Watanabe and Matsuda, 1966) and 2-2-IV (Bolton et al., 2010; Engelkes and Windels, 1996) are virulent on table beet and the closely related sugar beet (both Beta vulgaris L. ssp. vulgaris) and Rhizoctonia infections can cause severe yield losses in both the field and during processing (Gaskill, 1968; Hecker and Ruppel, 1977b; Ruppel et al., 1979; Stivers, n.d.). Sugar beet seedlings are susceptible to a wider range of AGs (Bolton et al., 2010; Rush et al., 1994; Windels and Nabben, 1989) compared with mature plants, resulting in damping off and stand reduction. In contrast, Windels and Nabben (1989) found that plants older than 8 weeks in their experiments were primarily infected by AG 2-2. This was corroborated by Bolton et al. ties. AGs 1, 2-2, and 4 infect beets, with AG
(2010) when they observed that out of a range of 15 tested AGs and SGs, only AG 2-2-IIIB and AG 2-2-IV caused significant disease in 10 -week-old sugar beet plants suggesting that mature plants are less susceptible to some AGs.

$R$. solani can affect beet plants at two stages in the crop's lifecycle: at the seedling stage and later in the growing season when plants are maturing (Sneh et al., 1996). Shortly after crop emergence, seedlings may damp-off caused by $R$. solani attacking the hypocotyl below the soil level. Severely diseased seedlings succumb, whereas mild cases result in less vigorous and misshapen mature roots (Sneh et al., 1996). The occurrence of $R$. solani on mature plants later in the growing season is often referred to as Rhizoctonia root and crown rot. In the case of table beets, the disease is commonly called pocket rot for the numerous empty "pockets" left behind in the field in the disease's wake (Pethybridge et al., 2018; Windels et al., 2009). Plants exhibit aboveground symptoms including black lesions on petioles touching the soil surface followed by the wilting and death of older leaves (Windels et al., 2009). Underground symptoms include copious circular root lesions ranging from 2 to $25 \mathrm{~mm}$ in diameter (Windels et al., 2009). Lesions have a well-defined border and exhibit alternating light and dark colors in concentric bands (Windels et al., 2009). Internally, roots may contain mycelium-filled cankers easily distinguished from healthy root tissue (Sneh et al., 1996). The fungus overwinters and survives for many years in colonized plant tissue and sclerotia (Abawi et al., 1986; Cubeta and Vilgalys, 1997). Sumner and Bell (1994) observed disease caused by AG 2-2 in crop plants and low soil inoculum densities (average of 3.8 colony-forming units per $100 \mathrm{~g}$ 32 months after planting) of that same subgroup in the third year of a study investigating survival of Rhizoctonia spp. in a rotation of a corn-snap bean double crop, peanut monocrop, and corn-snap bean double crop.

Current strategies for management of Rhizoctonia root and crown rot in beet include crop rotation with nonhost plants (Abawi et al., 1986; Hecker and Ruppel, 1976; Pethybridge et al., 2018), proper weed management (Harveson, 2003), avoiding covering plant crowns with soil during cultivation (Schneider et al., 1982), using treated seed (Abawi et al., 1986), and applying fungicides during the growing season (Colquhoun et al., 2020; Pethybridge et al., 2018). Growers often implement a combination of these strategies in an integrated pest management approach.

In 1977, Hecker and Ruppel (1977b) suggested a 4- to 5-year rotation of sugar beet with cereals, alfalfa, or maize to control $R$. solani. Many beet-growing areas list crop rotation as a management practice (Cattanach et al., 1991; Colquhoun et al., 2020; Khan et al., 2020; Stivers, n.d.). Management of this pathogen through crop rotation, however, has proven to be difficult because of the wide range of plants affected by AG 2-2. Decreasing effectiveness of suppression in table beet-maize rotations in New York led 
Ohkura et al. (2009) to investigate the situation. They found most of the isolates pathogenic on table beet tested were also pathogenic on maize. Isolates in AG 2-2, AG 5, and AG 11 were highly aggressive in the experiments (Ohkura et al., 2009). In 2011, Strausbaugh et al. observed that seven of the 18 AG 2-2 IIIB isolates from sugar beet tested in their study were capable of attacking maize and sugar beet. These findings supported previous work by Sumner and Bell (1982) in which AG 2-2 was discovered to induce lateral, brace, and crown rot in maize crops. In this study, AG 2-2 isolates from maize were highly virulent on other Georgian crops, including snap bean, lima bean, southernpea, juvenile soybean, and cucumber (Sumner and Bell, 1982). In spite of these findings, crop rotations are certainly not obsolete; without them, losses to the fungus would be much higher. In Nebraska, Schuster and Harris (1960) discovered 4 years of continuous sugar beets led to an average of $63 \%$ infected beets.

In-season fungicide applications are often used by growers to manage Rhizoctonia root and crown rot. Olaya et al. (1994) completed initial studies for chemical control of $R$. solani on table beet and since then several fungicides have been registered for use on beet (Arabiat and Khan, 2016). One of the most widely used fungicides, azoxystrobin, was labeled in 1999 and is effective for increasing stands and yields (Arabiat and Khan, 2016; Windels and Brantner, 2005) and controlling Rhizoctonia root and crown rot (Gugino et al., 2008; Kiewnick et al., 2001), but timing of application is crucial (Kirk et al., 2008; Stump et al., 2002). Fungicide applications can cost more than $\$ 60$

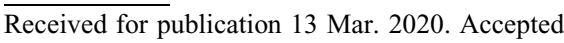
for publication 29 June 2020.

Published online 6 August 2020.

Partial support for this project was provided by the Midwest Food Products Association Grant No. M-3. We thank Linda Hanson, Michigan State University, U.S. Department of Agriculture (USDA)-Agricultural Research Station (ARS), for providing the R1 isolate and disease screening advice. We thank Lee Panella, USDA-ARS, for providing the resistant and susceptible sugar beet seed. We thank Barbara Hellier, USDA-ARS-National Plant Germplasm System, for providing the table beet seed. We thank Imad Eujayl, USDA, for providing the doubled haploid sugar beet line. We thank Amanda Gevens, Michael Havey, and anonymous peer reviewers for their critical and constructive review of the manuscript. We are grateful to Tedward Erker for statistical advice. Special thanks to Kristen Loria, Freddie Mildenhall, Matt Mirkes, and Taylor Sefzik for their assistance in disease evaluations.

Mention of a trademark, proprietary product, or vendor does not constitute a guarantee or warranty of the product by the University of Wisconsin-Madison and does not imply its approval to the exclusion of other products or vendors that also may be suitable.

I.L.G. is the corresponding author. E-mail: ilgoldma@ wisc.edu.

This is an open access article distributed under the CC BY-NC-ND license (https://creativecommons. org/licenses/by-nc-nd/4.0/). per hectare (M.F.R. Khan, personal communication). In addition to the challenge of timing applications and costs involved, the potential for $R$. solani populations to develop fungicide resistance is a threat to disease management (Jacobsen, 1997; Kiewnick et al., 2001). Several beet-growing areas have documented resistance in Cercospora beticola Sacc., another beet pathogen controlled by azoxystrobin (Abawi et al., 2014; Vaghefi et al., 2016) and resistance to azoxystrobin has been reported in other fungal pathogens affecting crops, including almond, grape, pistachio, strawberry, watermelon, and perennial ryegrass (Jiang et al., 2009; Ma et al., 2003; Stevenson et al., 2004; Vincelli and Dixon, 2002). Azoxystrobin-resistant isolates of $R$. solani AG 1-IA, the causal organism of rice sheath blight, were first reported in Louisiana in 2011, and studies showed that resistance had spread from the initial area reported and more than a third of the isolates collected exhibited resistance to the fungicide (Lunos, 2016; Olaya et al., 2012). Pethybridge et al. (2018) cautions the risk of azoxystrobin resistance development within R. solani. Arabiat and Khan (2016) investigated the sensitivity to fungicides of isolates of AG 2-2 collected from sugar beets before and after the registration and usage of fungicides to manage damping off and root and crown rot caused by $R$. solani. They found a mean half maximal effective concentration $\left(\mathrm{EC}_{50}\right)$ of $296.1 \mu \mathrm{g} \cdot \mathrm{mL}^{-1}$ for isolates after the registration and usage of azoxystrobin compared with an $\mathrm{EC}_{50}$ of $49.7 \mu \mathrm{g} \cdot \mathrm{mL}^{-1}$ before registration (Arabiat and Khan, 2016). Although azoxystrobin was still effective at the labeled rate in controlling $R$. solani in the greenhouse study, these results demonstrate the capability of Rhizoctonia populations to develop resistance to the fungicide.

A reduction in the effectiveness of crop rotation, the ability of $R$. solani to survive saprophytically in the soil for several years, and the threat of pathogen populations developing resistance to fungicides make developing host plant resistance critical to economically sustainable table beet production (Gaskill, 1968; Harveson et al., 2002; Pethybridge et al., 2018).

Efforts to breed B. vulgaris for resistance to $R$. solani have primarily focused on the close relative of the table beet, the sugar beet, by mass selection or recurrent field selection (Gaskill, 1968). Many Rhizoctonia-resistant sugar beet lines have been developed (Halloin et al., 2000; Hecker and Gaskill, 1972; Hecker and Ruppel, 1977a, 1985, 1988, 1991; Panella et al., 2015a) and screening is actively done in sugar beet breeding programs (Panella et al., 2015b). Hecker and Ruppel (1975) determined that resistance in sugar beet is polygenic, involving at least two loci, each with two or three alleles, and including modifying genes. They estimated a broad sense heritability of $\approx 0.65$ (Hecker and Ruppel, 1975). In conjunction with breeding for disease resistance, many publicly released $B$. vulgaris subsp. vulgaris sugar beet lines and accessions in the $\mathrm{Na}$ tional Plant Germplasm System (NPGS) have been evaluated for resistance to Rhizoctonia over the years (Hanson and Panella, 2007; Panella, 2000; Panella and Hanson, 2001; Panella and Ruppel, 1998; Panella et al., 2008).

The use of Rhizoctonia-resistant cultivars for sugar beet production is recommended. Harveson et al. (2002) recommended resistant cultivars as a practical and economical disease management approach, and in some cases the only effective approach (McGrath and Panella, 2019). Although resistance is available in sugar beet, it is incomplete and there is often decreased yield potential associated with resistant lines (Jacobsen et al., 2004). Planting resistant varieties may be less attractive, especially when they have shown responses to fungicide applications and still not achieved the yield of susceptible varieties with fungicide applications (Jacobsen, 2006).

One approach to breed for $R$. solani resistance in table beet germplasm might involve the introgression of resistance from sugar beet. However, progeny derived from crosses between sugar beet and table beet have exhibited external russeting and increased crown diameter (two distinctive characteristics of sugar beets) even after 10 generations of backcrossing (Wang and Goldman, 1999). These are undesirable qualities in the table beet industry. A different approach would be to identify resistance in existing table beet germplasm. At present, only $30 \%$ of NPGS table beet accessions have been evaluated for their responses to inoculation with $R$. solani [Germplasm Resources Information Network (GRIN), 2020].

Although resistance to Rhizoctonia root and crown rot has not been wellcharacterized in table beet, several cultivars have appeared on the market with putative resistance or tolerance. These include 'Shiraz', 'Rubra', 'Kestrel', 'Solo' (Pethybridge et al., 2018), and 'Pacemaker III' (Goldman, 1996; Pethybridge et al., 2018); however, these claims have not been experimentally verified. Despite these cultivars, little work has been accomplished in assessing reaction to $R$. solani in table beet and in breeding for host resistance. The goal of this study was to use controlled conditions in a greenhouse to evaluate the reaction of commercial cultivars, publicly available inbred table beet lines, and PIs from the U.S. NPGS to $R$. solani. We hypothesized that table beet accessions with different genetic backgrounds would respond differently to inoculation with R. solani AG 2-2.

\section{Materials and Methods}

Germplasm. A large pool of crop genetic diversity is available in the U.S. NPGS with more than 2000 B. vulgaris ssp. vulgaris accessions (GRIN, 2020). This includes both table beets used for processing and sugar beets for sugar production; however, only a small fraction, $\approx 200$ unique accessions, are 
vegetable types. Accessions with the production descriptor "type" and designation of "table" were identified through GRIN. In Nov. 2017 there were 234 accessions fitting that description. Duplicate and unavailable accessions were removed, and the 94 remaining accessions were used in this investigation (Table 1). This screen will be referred to as the "PI Screen." In addition to the PI Screen, 56 cultivars were identified from 23 commercial sources (Table 2). In addition, nine publicly available inbred lines released from the University of Wisconsin table beet breeding program were included, along with a recently released open-pollinated cultivar, Badger Flame, from the University of Wisconsin (Wisconsin Alumni Research Foundation, 2019). Efforts were made to obtain only nontreated seed, but several lines were available only with treated seed. 'Carillon', 'Eagle', and 'Vulture' were treated with thiram (Thiram 50WP, tetramethylthiuram disulfide; Bayer CropScience, Research Triangle Park, NC) and metalaxyl (Apron; Syngenta Crop Protection, LLC, Greensboro, NC). 'Pacemaker III' and 'Bohan' were treated with both thiram and metalaxyl as well as fludioxonil (Maxim4S; Syngenta Crop Protection, LLC). Of these three seed treatments, only fludioxonil is labeled to control seed decay, seedling damping off, and seedling blight caused by $R$. solani. Seed treatments are unlikely to interfere with fungal growth and infection by the time of inoculation (Jacobsen et al., 2004; Kataria and Gisi, 1996; Stump et al., 2004; Windels and Brantner, 2002). This screen is referred to as the "Commercial Screen."

To validate the results from the PI and Commercial Screens and quantify the amount of variation within accessions relative to the greenhouse environment itself, a third screen was conducted. This screen included a subset of several accessions from both the PI and Commercial Screens, along with the doubled haploid sugar beet line KDH13 (Eujayl et al., 2016) (Table 3). This screen made use of increased replication compared with the PI and Commercial Screens and is referred to as the "Validation Screen." In each of the three screens, the table beet inbred line W364 was included as a control because it was fairly resistant to Rhizoctonia in our pilot screens (K. Wigg and I. Goldman, unpublished data). The inbred line W364 is a commonly used parent for $\mathrm{F}_{1}$ hybrid table beet cultivars (Goldman, 1996). Sugar beet inbred lines FC709-2 and FC901/C817 were also included as resistant and susceptible controls, respectively (Gaskill et al., 1967; Panella, 1999) [courtesy of Lee Panella, Crops Research Unit, U.S. Department of Agriculture-Agricultural Research Station (USDA-ARS)]. The Sugar Beet Research Unit in Ft. Collins, CO, uses FC709-2 (Panella, 1999) as a highly resistant control and FC901/C817 (Fenwick et al., 2018; Gaskill et al., 1967; Panella and Hanson, 2001) as a susceptible control in their Rhizoctonia screens. All germplasms, whether inbreds, PI accessions, or commer- cial lines, are hereafter referred to as accessions.

Greenhouse culture. All screens were completed in the Walnut Street Greenhouses at the University of Wisconsin-Madison. Experiments were conducted from Nov. 2017 through Jan. 2019. A minimum of five seeds were sown into a 3:1 mix (by volume) of silty loam compost soil collected from West Madison Agricultural Research Station (Arlington, WI) and soilless medium (MetroMix; Sun Gro Horticulture, Agawam, MA) in 2780 $\mathrm{cm}^{3}$ plastic pots. Following germination and plant establishment, each pot was thinned to two plants per pot. High-pressure sodium supplemental lighting $(1000 \mu \mathrm{mol}$ at bench height) provided 16-h daylengths. Airconditioned greenhouses with forced-air heating were used to maintain temperatures between 25 and $30^{\circ} \mathrm{C}$ to provide a conducive environment for $R$. solani disease development (Parmeter, 1970). Beets were watered as needed daily for optimum plant growth. A $400-\mathrm{mg} \cdot \mathrm{L}^{-1}$ solution of $20 \mathrm{~N}-4.4 \mathrm{P}-16.6 \mathrm{~K}$ fertilizer with micronutrients (Peters Professional Peat-Lite Special; ICL Specialty Fertilizers, Dublin, OH) was applied as needed for optimum plant growth. Bacillus thuringiensis ssp. israelensis $35 \%$ to $45 \%$ (Gnatrol WDG; Valent Biosciences Corporation, Libertyville, IL) was applied as needed to manage fungus gnats.

Because of the endemic nature of fungus gnats in the greenhouse environment, along with the added attraction of fungus from the inoculation of $R$. solani in our experiment (Cloyd, 2010), fungus gnats were present in our experiments and we included their presence/absence in our analysis. When disease symptoms other than those caused by $R$. solani were present, we included their presence/absence in our analysis and referred to these as "other diseases."

Experimental design. Each screen was replicated in time and conducted twice. The term replicate is used here to describe each experimental run but is also used to describe a complete set of treatments within a run. Following planting, pots were placed into a randomized complete block design with each block assigned to the 3 - or 5-week postinoculation evaluation period. In both the PI and Commercial Screens, each accession was represented by eight plants across the two replicates and two plants in each evaluation period. Both the PI and Commercial Screens included FC901/C817, FC709-2, and W364B as noninoculated controls. In the Validation Screen, each accession was represented by 40 plants across the two replicates and 10 plants in each evaluation period. In addition to the aforementioned three controls, the Validation Screen also included W411B as a noninoculated control. W411B was included as a noninoculated control because it exhibited little variation and was susceptible in the Commercial Screen.

Inoculations. All inoculations were performed using $R$. solani isolate R-1 (AG-2-2IIIB) obtained from Linda Hanson, USDA-ARS (Nagendran et al., 2009). The isolate was grown on autoclaved barley grains to produce the inoculum and was stored at 4 to $7{ }^{\circ} \mathrm{C}$ until use according to Naito et al. (1993). The isolate used in this study was originally collected from an infected sugar beet root from Platteville, CO, in the 1960s. Tests conducted by Michigan State researchers have classified the isolate as highly virulent on $B$. vulgaris ssp. vulgaris (L. Hanson, personal communication; Nagendran et al., 2009). This barleyfungus inoculum was roughly ground using a coffee grinder (FreshGrind; Hamilton Beach Brands, Glen Allen, VA) immediately before artificial inoculation. Each plant was inoculated by displacing a small amount of growth medium adjacent to the root 2 to 3 $\mathrm{cm}$ below the soil surface and depositing $6 \mathrm{~g}$ or $\approx 6 \mathrm{~mL}$ of ground inoculum (Hecker and Ruppel, 1977b; A. Fenwick, personal communication). Growth medium was then smoothed back over the inoculum. Plants were gently watered immediately following inoculations and then watered daily.

Disease testing. Artificial inoculation took place 8 weeks after planting. Noninoculated controls were included in each block and replication. Plants were evaluated for disease at 3 and 5 weeks postinoculation. The timeline of this screen was in accordance with sugar beet screening methods (L. Panella, personal communication).

At the time of evaluation, plants were gently removed from the medium. The beet tops were separated from the roots at the crown using a knife, weighed, and then discarded. Roots were brushed off with a cloth, gently washed with tap water, dried with a cloth, and then weighed. A visual disease rating scale adapted from the visual scale described by Campbell et al. (2014) and first used by Ruppel et al. (1979) was used to assign an external and internal disease rating for each plant (Fig. 1). Following the external rating, each root was halved from the crown to the bottom. The tissue visible from this cut was used to assign an internal rating. Ratings ranged from 0 to 5 , with a rating of $0=0 \%$ diseased tissue, root surface clean with no visible lesions; $1=1 \%$ to $10 \%$ diseased tissue, superficial, scattered nonactive lesions; $2=$ $11 \%$ to $30 \%$ of root affected; $3=31 \%$ to $60 \%$ of root affected; $4=61 \%$ to $99 \%$ of root blackened with rot extending into interior; and $5=$ root $100 \%$ rotted and foliage was dead or dying (adapted from Campbell et al., 2014). These groupings were chosen because the researcher could visually distinguish among them.

To normalize the data for linear regression, the internal and external disease ratings for each plant were converted into a diseased tissue percentage based on the following: $0=$ $0 \% ; 1=5.5 \% ; 2=20.5 \% ; 3=45.5 \% ; 4=$ $80 \%$; and $5=100 \%$. The diseased tissue percentages for each of the ratings were determined based on the mean value within that rating. For example, a rating of 3 ranges from $31 \%$ to $60 \%$ diseased tissue, so the mean value would be $45.5 \%$. To give each root a single diseased tissue percentage, those 
Table 1. Mean diseased tissue percentage, most likely internal disease ratings and probabilities, and disease progression categories of 97 Beta vulgaris subsp. vulgaris PI accessions in response to inoculation with Rhizoctonia solani AG 2-2 isolate R1 in a greenhouse screen evaluated in 2018.

\begin{tabular}{|c|c|c|c|c|c|c|c|c|c|}
\hline Code $^{z}$ & Accession & Name & Origin $^{y}$ & $\begin{array}{l}\text { Mean diseased } \\
\text { tissue } \\
\text { percentage }^{\mathrm{x}}\end{array}$ & $\begin{array}{c}\text { First most } \\
\text { likely } \\
\text { internal DR }\end{array}$ & $\begin{array}{c}\text { Second most } \\
\text { likely } \\
\text { internal DR } \\
\end{array}$ & $\begin{array}{c}\text { Probability } \\
\text { of first most likely } \\
\text { internal DR (SE) }\end{array}$ & $\begin{array}{c}\text { Probability of } \\
\text { second most } \\
\text { likely internal DR }(\mathrm{sE})^{\mathrm{w}}\end{array}$ & $\begin{array}{c}\text { Disease } \\
\text { progression }^{v}\end{array}$ \\
\hline$\overline{68}$ & Ames 22164 & $\begin{array}{l}\text { Long Smoothe } \\
\text { Dark Red }\end{array}$ & US & $1.9 \mathrm{a}$ & 0 & 1 & $0.56(0.20,0.91)$ & $0.37(0.15,0.59)$ & $\mathrm{SC}$ \\
\hline 86 & PI 169015 & IDBBNR 5260 & TK & $6.4 \mathrm{ab}$ & 1 & 0 & $0.46(0.37,0.54)$ & $0.37(0.11,0.64)$ & $\mathrm{SC}$ \\
\hline 73 & PI 105335 & Tzu Lo Pu Tou & China & $6.6 \mathrm{ab}$ & 1 & 2 & $0.47(0.42,0.52)$ & $0.27(0.10,0.45)$ & $\mathrm{SC}$ \\
\hline 47 & PI 181717 & IDBBNR 5359 & LB & $10.0 \mathrm{ab}$ & 1 & 2 & $0.46(0.37,0.54)$ & $0.32(0.15,0.49)$ & $\mathrm{SC}$ \\
\hline 93 & PI 148625 & Chaghonda & Iran & $10.9 \mathrm{ab}$ & 1 & 2 & $0.46(0.37,0.54)$ & $0.32(0.14,0.50)$ & $\mathrm{SC}$ \\
\hline 53 & PI 116906 & Lablabu & $\mathrm{AF}$ & $12.1 \mathrm{abc}$ & 1 & 2 & $0.40(0.26,0.54)$ & $0.40(0.26,0.54)$ & $\mathrm{SC}$ \\
\hline 41 & PI 323938 & Avon Early & UK & $12.2 \mathrm{abc}$ & 1 & 0 & $0.45(0.36,0.55)$ & $0.38(0.12,0.63)$ & $\mathrm{SC}$ \\
\hline 65 & NSL 28024 & Extra Early & US & $13.1 \mathrm{abcd}$ & 1 & 0 & $0.47(0.41,0.53)$ & $0.31(0.07,0.56)$ & $\mathrm{SC}$ \\
\hline 89 & PI 169028 & IDBBNR 5272 & TK & 13.1 abcde & 1 & 2 & $0.42(0.29,0.54)$ & $0.38(0.23,0.53)$ & $\mathrm{SC}$ \\
\hline 46 & PI 179175 & IDBBNR 5347 & Iraq & 13.2 abcde & 0 & 1 & $0.45(0.18,0.72)$ & $0.43(0.30,0.55)$ & SS \\
\hline 94 & PI 590606 & Fordhook Giant & US & 13.3 abcdef & 1 & 2 & $0.41(0.27,0.54)$ & $0.39(0.25,0.54)$ & $\mathrm{SC}$ \\
\hline 3 & PI 177272 & IDBBNR 5338 & TK & 14.2 abcdef & 1 & 0 & $0.47(0.43,0.52)$ & $0.28(0.06,0.49)$ & $\mathrm{SC}$ \\
\hline 59 & PI 380754 & IDBBNR 9582 & Iran & 14.4 abcdef & 1 & 2 & $0.45(0.36,0.54)$ & $0.33(0.16,0.50)$ & SS \\
\hline 37 & PI 271439 & IDBBNR 5435 & India & 14.7 abcdef & 1 & 2 & $0.47(0.42,0.52)$ & $0.27(0.10,0.45)$ & $\mathrm{SC}$ \\
\hline 7 & W6 22192 & POL 175453 & PL & 15.0 abcdef & 1 & 2 & $0.42(0.29,0.54)$ & $0.38(0.24,0.53)$ & $\mathrm{SC}$ \\
\hline 74 & PI 109039 & IDBBNR 5161 & TK & 15.2 abcdef & 1 & 2 & $0.47(0.41,0.53)$ & $0.29(0.12,0.46)$ & SS \\
\hline 42 & PI 357351 & Polsko & $\mathrm{MC}$ & 15.7 abcdef & 1 & 2 & $0.47(0.40,0.53)$ & $0.29(0.12,0.47)$ & SS \\
\hline 75 & PI 124528 & Chakunda & India & 15.9 abcdef & 1 & 0 & $0.47(0.43,0.51)$ & $0.26(0.05,0.47)$ & $\mathrm{SC}$ \\
\hline 16 & PI 612334 & Rodbeta Slowbolt & SW & 16.7 abcdef & 2 & 1 & $0.42(0.30,0.55)$ & $0.37(0.22,0.53)$ & SS \\
\hline 51 & W6 44532 & Feuer Kugel & $\mathrm{CN}$ & 17.0 abcdef & 1 & 2 & $0.44(0.34,0.54)$ & $0.35(0.18,0.51)$ & SS \\
\hline 25 & PI 531261 & Detroit & $\mathrm{HN}$ & 17.1 abcdef & 1 & 2 & $0.42(0.30,0.54)$ & $0.38(0.23,0.53)$ & SS \\
\hline 26 & PI 590593 & $\begin{array}{l}\text { Improved } \\
\text { Early Egyptian }\end{array}$ & NI & 17.5 abcdef & 2 & 1 & $0.47(0.41,0.53)$ & $0.28(0.11,0.45)$ & $\mathrm{SC}$ \\
\hline 39 & PI 285591 & $\begin{array}{l}\text { Okragly } \\
\text { Ciemnoczerwony }\end{array}$ & PL & 17.7 abcdef & 1 & 2 & $0.45(0.35,0.54)$ & $0.34(0.17,0.51)$ & SS \\
\hline 81 & PI 163178 & Choghundur & India & 19.7 abcdef & 2 & 1 & $0.41(0.28,0.55)$ & $0.39(0.24,0.53)$ & SS \\
\hline 80 & PI 144675 & IDBBNR 5229 & Iran & 20.1 abcdef & 1 & 2 & $0.42(0.29,0.54)$ & $0.38(0.23,0.53)$ & $\mathrm{SC}$ \\
\hline 11 & PI 612339 & Albina Vereduna & NT & 20.2 abcdef & 1 & 2 & $0.44(0.34,0.54)$ & $0.34(0.18,0.51)$ & SS \\
\hline 13 & PI 612337 & Kogel & NT & 20.4 abcdef & 2 & 1 & $0.46(0.38,0.54)$ & $0.32(0.15,0.48)$ & $\mathrm{SC}$ \\
\hline 28 & PI 590600 & Detroit Perfected & NI & 20.4 abcdef & 1 & 0 & $0.47(0.43,0.51)$ & $0.26(0.06,0.47)$ & $\mathrm{SC}$ \\
\hline 1 & PI 169032 & IDBBNR 5276 & $\mathrm{TK}$ & 20.4 abcdef & 1 & 2 & $0.44(0.35,0.54)$ & $0.34(0.18,0.50)$ & $\mathrm{SC}$ \\
\hline 72 & NSL 6611 & Eclipse & US & 20.4 abcdef & 1 & 2 & $0.45(0.37,0.54)$ & $0.32(0.16,0.49)$ & $\mathrm{SC}$ \\
\hline 95 & FC $901 / C 817^{\mathrm{t}}$ & FC901/C817 & US & 20.5 abcdef & 1 & 0 & $0.47(0.43,0.51)$ & $0.29(0.12,0.46)$ & $\mathrm{SC}$ \\
\hline 67 & Ames 8292 & IDBBNR 9509 & UK & 20.5 abcdef & 2 & 1 & $0.42(0.29,0.54)$ & $0.38(0.24,0.53)$ & $\mathrm{SC}$ \\
\hline 71 & NSL 6320 & Winter Keeper & NI & 20.5 abcdef & 2 & 1 & $0.46(0.39,0.54)$ & $0.31(0.14,0.47)$ & $\mathrm{SC}$ \\
\hline 32 & PI 596529 & Bordo 237 & AR & 21.1 abcdef & 2 & 1 & $0.41(0.27,0.54)$ & $0.39(0.25,0.54)$ & SS \\
\hline 49 & PI 177275 & Beledi & $\mathrm{TK}$ & 21.2 abcdef & 2 & 1 & $0.42(0.29,0.55)$ & $0.38(0.23,0.53)$ & $\mathrm{SC}$ \\
\hline 87 & PI 169019 & IDBBNR 5264 & TK & 21.5 abcdef & 1 & 2 & $0.43(0.31,0.54)$ & $0.37(0.21,0.52)$ & $\mathrm{SC}$ \\
\hline 33 & PI 612328 & Bikores & NT & 21.5 abcdef & 2 & 1 & $0.47(0.40,0.54)$ & $0.30(0.13,0.46)$ & $\mathrm{SC}$ \\
\hline 84 & PI 164805 & Choghundar & India & 21.6 abcdef & 2 & 3 & $0.47(0.42,0.53)$ & $0.22(0.10,0.34)$ & $\mathrm{SC}$ \\
\hline 6 & W6 22468 & BV-00-01 & $\mathrm{SL}$ & 21.6 abcdef & 1 & 2 & $0.44(0.33,0.54)$ & $0.35(0.19,0.51)$ & $\mathrm{SC}$ \\
\hline 15 & PI 612336 & Albino White & $\mathrm{CN}$ & 22.1 abcdef & 1 & 2 & $0.45(0.35,0.54)$ & $0.34(0.17,0.50)$ & $\mathrm{SC}$ \\
\hline 18 & PI 612330 & Replata & NT & 22.1 abcdef & 1 & 2 & $0.42(0.30,0.54)$ & $0.37(0.22,0.53)$ & SS \\
\hline 83 & PI 164659 & IDBBNR 5245 & India & 22.1 abcdef & 2 & 1 & $0.40(0.27,0.54)$ & $0.40(0.26,0.54)$ & SS \\
\hline 88 & PI 169022 & IDBBNR 5267 & $\mathrm{TK}$ & 22.1 abcdef & 2 & 1 & $0.41(0.29,0.54)$ & $0.39(0.24,0.53)$ & $\mathrm{SC}$ \\
\hline 85 & PI 164968 & IDBBNR 5251 & $\mathrm{TK}$ & 22.3 abcdef & 2 & 1 & $0.44(0.32,0.55)$ & $0.36(0.20,0.52)$ & $\mathrm{SC}$ \\
\hline 23 & PI 414934 & IDBBNR 4621 & SA & 22.4 abcdef & 2 & 1 & $0.44(0.33,0.55)$ & $0.36(0.20,0.51)$ & $\mathrm{SC}$ \\
\hline 91 & PI 222234 & IDBBNR 5391 & Iran & 22.6 abcdef & 1 & 2 & $0.46(0.38,0.54)$ & $0.31(0.14,0.48)$ & $\mathrm{SC}$ \\
\hline 14 & PI 612342 & $\begin{array}{l}\text { Rote Kugel } \\
\text { Sperlings Bluta }\end{array}$ & $\mathrm{DE}$ & 23.1 abcdef & 2 & 1 & $0.45(0.35,0.55)$ & $0.34(0.18,0.50)$ & SS \\
\hline 63 & Ames 14432 & Bordo- 60 & BG & 23.1 abcdef & 1 & 2 & $0.41(0.28,0.54)$ & $0.39(0.24,0.53)$ & $\mathrm{SC}$ \\
\hline 90 & PI 169029 & Pancar & $\mathrm{TK}$ & 23.9 abcdef & 1 & 2 & $0.45(0.37,0.54)$ & $0.32(0.16,0.49)$ & $\mathrm{SC}$ \\
\hline
\end{tabular}


Table 1. (Continued) Mean diseased tissue percentage, most likely internal disease ratings and probabilities, and disease progression categories of 97 Beta vulgaris subsp. vulgaris PI accessions in response to inoculation with Rhizoctonia solani AG 2-2 isolate R1 in a greenhouse screen evaluated in 2018.

\begin{tabular}{|c|c|c|c|c|c|c|c|c|c|}
\hline $\operatorname{Code}^{\mathrm{z}}$ & Accession & Name & Origin ${ }^{y}$ & $\begin{array}{l}\text { Mean diseased } \\
\text { tissue } \\
\text { percentage }^{\mathrm{x}}\end{array}$ & $\begin{array}{c}\text { First most } \\
\text { likely } \\
\text { internal DR }{ }^{\mathrm{w}}\end{array}$ & $\begin{array}{l}\text { Second most } \\
\text { likely } \\
\text { internal } \mathrm{DR}^{\mathrm{w}}\end{array}$ & $\begin{array}{c}\text { Probability } \\
\text { of first most likely } \\
\text { internal DR (SE) }\end{array}$ & $\begin{array}{c}\text { Probability of } \\
\text { second most } \\
\text { likely internal DR }(\mathrm{SE})^{\mathrm{w}}\end{array}$ & $\begin{array}{c}\text { Disease } \\
\text { progression }^{v}\end{array}$ \\
\hline$\overline{38}$ & PI 285590 & Epipski Hoser & PL & 24.3 abcdef & 2 & 1 & $0.47(0.41,0.53)$ & $0.28(0.12,0.45)$ & SS \\
\hline 40 & PI 296129 & IDBBNR 5459 & US & 25.3 abcdef & 2 & 1 & $0.45(0.36,0.54)$ & $0.33(0.17,0.49)$ & $\mathrm{SC}$ \\
\hline 60 & PI 140353 & IDBBNR 5203 & Iran & 25.4 abcdef & 1 & 2 & $0.45(0.36,0.54)$ & $0.34(0.18,0.50)$ & $\mathrm{SC}$ \\
\hline 52 & PI 222233 & Laboo & Iran & 25.7 abcdef & 2 & 1 & $0.41(0.27,0.54)$ & $0.39(0.25,0.54)$ & $\mathrm{SC}$ \\
\hline 44 & PI 357360 & Ohridska Zolta & $\mathrm{MC}$ & 25.8 abcdef & 1 & 2 & $0.45(0.35,0.54)$ & $0.34(0.17,0.50)$ & SS \\
\hline 69 & NSL 28026 & Gardeners Model & US & 26.2 abcdef & 2 & 1 & $0.46(0.37,0.54)$ & $0.32(0.16,0.49)$ & SS \\
\hline 4 & W6 44530 & Golden Beet & $\mathrm{CN}$ & 26.2 abcdef & 2 & 1 & $0.48(0.43,0.52)$ & $0.23(0.07,0.39)$ & $\mathrm{SC}$ \\
\hline 36 & PI 269310 & Spangsbjerg & SW & 26.3 abcdef & 2 & 1 & $0.46(0.39,0.54)$ & $0.30(0.14,0.47)$ & SS \\
\hline 9 & W6 22161 & POL 175449 & PL & 26.5 abcdef & 2 & 1 & $0.41(0.27,0.54)$ & $0.39(0.25,0.53)$ & $\mathrm{SC}$ \\
\hline 21 & PI 590635 & $\begin{array}{l}\text { Burpees Improved } \\
\text { Blood Turnip }\end{array}$ & $\mathrm{NI}$ & 27.2 abcdef & 2 & 1 & $0.46(0.38,0.54)$ & $0.32(0.15,0.48)$ & $\mathrm{SC}$ \\
\hline 5 & W6 44528 & Bull's Blood & $\mathrm{CN}$ & 27.3 abcdef & 2 & 1 & $0.46(0.38,0.54)$ & $0.32(0.15,0.48)$ & SS \\
\hline 64 & Ames 22163 & Iowa & US & 27.5 abcdef & 2 & 3 & $0.47(0.40,0.53)$ & $0.23(0.11,0.35)$ & SS \\
\hline 2 & PI 174059 & IDBBNR 5310 & TK & 27.8 abcdef & 2 & 1 & $0.44(0.34,0.55)$ & $0.35(0.19,0.50)$ & $\mathrm{SC}$ \\
\hline 17 & PI 612332 & Egyptische Egy & NT & 27.9 abcdef & 2 & 1 & $0.47(0.40,0.53)$ & $0.29(0.13,0.46)$ & SS \\
\hline 35 & PI 269309 & $\begin{array}{l}\text { Good for } \\
\text { All Rikssort }\end{array}$ & SW & 28.1 abcdef & 2 & 1 & $0.47(0.41,0.53)$ & $0.28(0.12,0.45)$ & $\mathrm{SC}$ \\
\hline 58 & PI 357356 & Okruglo & $\mathrm{MC}$ & 28.8 abcdef & 2 & 1 & $0.45(0.36,0.55)$ & $0.33(0.17,0.49)$ & SS \\
\hline 20 & PI 590627 & Special Crosby & $\mathrm{NI}$ & 28.8 abcdef & 2 & 1 & $0.48(0.43,0.52)$ & $0.24(0.08,0.40)$ & $\mathrm{SC}$ \\
\hline 31 & PI 592989 & Red Baron & US & 29.0 abcdef & 2 & 1 & $0.45(0.36,0.54)$ & $0.33(0.17,0.49)$ & $\mathrm{SC}$ \\
\hline 79 & PI 142820 & Choghondar & Iran & 29.1 abcdef & 2 & 1 & $0.43(0.31,0.56)$ & $0.37(0.20,0.53)$ & $\mathrm{SC}$ \\
\hline 29 & PI 590636 & $\begin{array}{l}\text { Early Wonder/ } \\
\text { Boston Crosby }\end{array}$ & NI & 30.1 abcdef & 2 & 1 & $0.48(0.43,0.53)$ & $0.26(0.09,0.42)$ & $\mathrm{SC}$ \\
\hline 70 & NSL 43404 & Parma Globe & NI & 30.3 abcdef & 1 & 2 & $0.41(0.28,0.54)$ & $0.39(0.24,0.54)$ & $\mathrm{SC}$ \\
\hline 78 & PI 142810 & Choghondar & Iran & 30.6 abcdef & 1 & 2 & $0.41(0.28,0.54)$ & $0.39(0.24,0.54)$ & $\mathrm{SC}$ \\
\hline 43 & PI 357357 & Okrugla & $\mathrm{MC}$ & 31.1 abcdef & 2 & 1 & $0.44(0.33,0.55)$ & $0.35(0.19,0.51)$ & SS \\
\hline 19 & PI 612331 & Detroit Vuurbal & NT & 32.1 abcdef & 2 & 1 & $0.44(0.33,0.55)$ & $0.36(0.20,0.51)$ & QP \\
\hline 34 & PI 612329 & Regala & NT & 32.6 bcdef & 2 & 1 & $0.47(0.42,0.53)$ & $0.27(0.11,0.44)$ & $\mathrm{SC}$ \\
\hline 10 & PI 612340 & Boltardy & NT & 33.7 bcdef & 2 & 3 & $0.47(0.42,0.53)$ & $0.22(0.10,0.34)$ & $\mathrm{SC}$ \\
\hline 30 & PI 590640 & $\begin{array}{l}\text { Detroit Dark } \\
\text { Red Garnet }\end{array}$ & $\mathrm{NI}$ & 34.3 bcdef & 2 & 1 & $0.48(0.43,0.52)$ & $0.26(0.09,0.42)$ & SS \\
\hline 97 & W364B ${ }^{s}$ & W364B & US & $40.5 \mathrm{cdef}$ & 1 & 2 & $0.44(0.36,0.52)$ & $0.35(0.24,0.47)$ & $\mathrm{SC}$ \\
\hline 82 & PI 164292 & IDBBNR 5239 & India & $43.0 \mathrm{def}$ & 2 & 3 & $0.47(0.42,0.52)$ & $0.22(0.10,0.34)$ & $\mathrm{SC}$ \\
\hline 27 & PI 590595 & Eastern Wonder & $\mathrm{NI}$ & $43.6 \mathrm{ef}$ & 2 & 3 & $0.44(0.33,0.54)$ & $0.28(0.17,0.39)$ & QP \\
\hline 56 & PI 103042 & Ken Ta Tsai & NI & $43.8 \mathrm{f}$ & 2 & 1 & $0.47(0.40,0.54)$ & $0.29(0.13,0.46)$ & SS \\
\hline
\end{tabular}

${ }^{\mathrm{z}}$ Code refers to the accessions in Supplemental Fig. 2 and Supplemental Table 2.

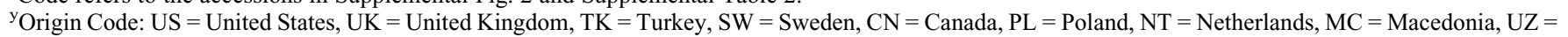
Uzbekistan, $\mathrm{LB}=$ Lebanon, $\mathrm{HN}=$ Hungary, $\mathrm{AR}=$ Armenia, $\mathrm{BG}=$ Bulgaria, $\mathrm{AF}=$ Afghanistan, $\mathrm{DN}=\mathrm{Denmark}$, $\mathrm{SU}=\mathrm{Soviet} \mathrm{Union}, \mathrm{ET}=\mathrm{Ethiopia}, \mathrm{SL}=\mathrm{Sri}$ Lanka, $\mathrm{SA}=$ South Africa, DE = Germany, NI = Not Included on Germplasm Resources Information Network.

$\mathrm{x}_{\mathrm{HSD}_{0.05}}=6.2$. Different letters indicate significant differences as determined by Tukey pairwise comparisons at $\alpha=0.05$.

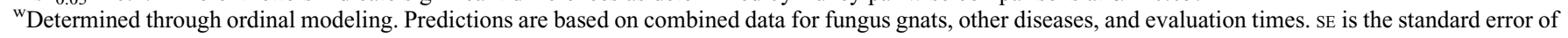
the probability of each most likely disease rating (DR).

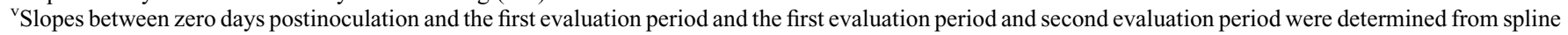

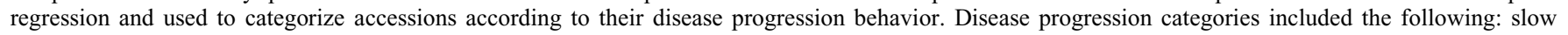

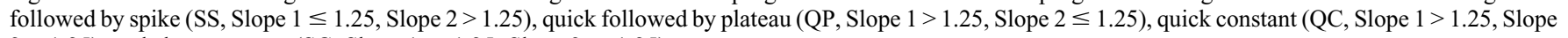
$2>1.25$ ), and slow constant (SC, Slope $1 \leq 1.25$, Slope $2 \leq 1.25$ ).

${ }^{\mathrm{u}} \mathrm{FC} 709-2$, resistant sugar beet control.

${ }^{\mathrm{t}} \mathrm{FC}$ 901/C817, susceptible sugar beet control.

${ }^{\mathrm{s}} \mathrm{W} 364 \mathrm{~B}$, table beet inbred control.

percentages were then weighted as described by the following equation:

(External Diseased Tissue Percentage $* 0.25$ )

$+($ Internal Diseased Tissue Percentage * 0.75)

= Weighted Average Diseased Tissue Percentage

The weighted average was calculated to emphasize the internal root characteristics because those are of greater importance for processing beets (A. Bennett, Seneca Foods, personal communication). External lesions, which are closer to the root surface, can be peeled in processing without much loss of tissue (M. Badtke, Del Monte Foods, Inc., personal communication). Following the calculation of the weighted average diseased tissue percentage for one plant, the percentages for the two plants in a pot were averaged as subsamples, providing one averaged weighted diseased tissue percentage for a single pot. These averages were then used for linear regression analysis and to calculate the area under the disease progression curve (AUDPC) (Madden et al., 2007). R code from Schandry (2017) was adapted to provide areas under the curve for each accession. Additional notes were collected regarding the presence of fungus gnat larvae and other diseases.

Statistical analysis. R statistical software (R 3.5.2; R Core Team, 2018) was used for all analyses ( $\mathrm{R}$ code available from authors by request). Analysis of variance (ANOVA) was conducted using the "stats" package in R (R Core Team, 2018). Linear models were fitted using the "ImerTest" package (Kuznetsova et al., 2017). Models were fitted first for the Validation Screen because that experiment included the highest number of samples per accession. The model for the Validation Screen included accession, inoculation status, evaluation time ( 3 and 5 weeks postinoculation), presence of fungus gnats, and presence of other diseases as fixed effects. Models were selected using the Akaike information criterion provided by the "stepAIC" function in the "MASS" package (Venables and Ripley, 2002) in combination with researcher discretion. ANOVAs were conducted for the weighted average diseased tissue percentages to evaluate the significance of differences in the level of resistance among the genotypes. Differences among accessions in the presence of $R$. solani were evaluated with the noninoculated controls excluded from the dataset. Following all significant ANOVAs $(P<0.05)$, Tukey's 
Table 2. Mean diseased tissue percentage, most likely internal disease ratings and probabilities, and disease progression categories of 62 Beta vulgaris subsp. vulgaris commercial cultivars and publicly available inbred accessions in response to inoculation with Rhizoctonia solani AG 2-2 isolate R1 in a greenhouse screen evaluated in 2018.

\begin{tabular}{|c|c|c|c|c|c|c|c|}
\hline $\operatorname{Code}^{\mathrm{z}}$ & Accession & $\begin{array}{l}\text { Mean diseased } \\
\text { tissue percentage }\end{array}$ & $\begin{array}{c}\text { First most } \\
\text { likely internal } \mathrm{DR}^{\mathrm{x}}\end{array}$ & $\begin{array}{l}\text { Second most likely } \\
\text { internal } \mathrm{DR}^{\mathrm{x}}\end{array}$ & $\begin{array}{c}\text { Probability of } \\
\text { first most likely } \\
\text { internal DR }(\mathrm{SE})^{\mathrm{x}}\end{array}$ & $\begin{array}{c}\text { Probability of } \\
\text { second most likely } \\
\text { internal DR }(\mathrm{SE})^{\mathrm{x}}\end{array}$ & $\begin{array}{c}\text { Disease } \\
\text { progression }\end{array}$ \\
\hline$\overline{64}$ & FC709-2 & $6.6 \mathrm{a}$ & 1 & 0 & $0.49(0.41,0.57)$ & $0.31(0.06,0.55)$ & $\mathrm{SC}$ \\
\hline 28 & Long Season & $16.3 \mathrm{ab}$ & 1 & 2 & $0.48(0.37,0.58)$ & $0.32(0.15,0.49)$ & $\mathrm{SC}$ \\
\hline 12 & Red Cloud & $16.7 \mathrm{ab}$ & 1 & 2 & $0.41(0.26,0.57)$ & $0.40(0.26,0.55)$ & $\mathrm{SC}$ \\
\hline 52 & Albina Vereduna & $19.2 \mathrm{ab}$ & 2 & 1 & $0.43(0.31,0.55)$ & $0.38(0.21,0.55)$ & $\mathrm{SC}$ \\
\hline 65 & $\mathrm{~W} 364 \mathrm{~B}^{\mathrm{u}}$ & $21.9 \mathrm{ab}$ & 1 & 0 & $0.47(0.34,0.59)$ & $0.40(0.14,0.65)$ & $\mathrm{SC}$ \\
\hline 17 & Bull's Blood & $22.1 \mathrm{ab}$ & 1 & 2 & $0.46(0.34,0.58)$ & $0.34(0.18,0.51)$ & SS \\
\hline 11 & Boro & $23.0 \mathrm{ab}$ & 2 & 3 & $0.47(0.38,0.55)$ & $0.29(0.14,0.45)$ & $\mathrm{SC}$ \\
\hline 33 & Perfected Detroit & $23.0 \mathrm{ab}$ & 1 & 2 & $0.47(0.37,0.58)$ & $0.32(0.15,0.49)$ & QP \\
\hline 10 & Falcon & $23.6 \mathrm{ab}$ & 1 & 2 & $0.49(0.42,0.57)$ & $0.27(0.10,0.44)$ & $\mathrm{SC}$ \\
\hline 47 & Carillon & $23.9 \mathrm{ab}$ & 2 & 1 & $0.44(0.33,0.56)$ & $0.37(0.20,0.53)$ & $\mathrm{SC}$ \\
\hline 45 & Green Top Bunching & $24.4 \mathrm{ab}$ & 2 & 1 & $0.46(0.38,0.55)$ & $0.32(0.15,0.50)$ & $\mathrm{SC}$ \\
\hline 51 & Sweetheart & $25.5 \mathrm{ab}$ & 2 & 1 & $0.46(0.37,0.55)$ & $0.33(0.16,0.50)$ & $\mathrm{SC}$ \\
\hline 61 & Badger Flame & $25.6 \mathrm{ab}$ & 2 & 1 & $0.46(0.38,0.55)$ & $0.32(0.14,0.49)$ & $\mathrm{SC}$ \\
\hline 53 & W443B & $25.7 \mathrm{ab}$ & 2 & 1 & $0.48(0.41,0.54)$ & $0.28(0.09,0.47)$ & QP \\
\hline 56 & W427B & $25.9 \mathrm{ab}$ & 2 & 1 & $0.48(0.41,0.54)$ & $0.28(0.10,0.46)$ & $\mathrm{SC}$ \\
\hline 21 & Babybeat & $26.0 \mathrm{ab}$ & 2 & 1 & $0.48(0.42,0.53)$ & $0.27(0.10,0.45)$ & SS \\
\hline 3 & Bohan & $26.1 \mathrm{ab}$ & 2 & 1 & $0.47(0.40,0.54)$ & $0.30(0.12,0.48)$ & QP \\
\hline 63 & $\mathrm{FC} 901 / \mathrm{C} 817^{\mathrm{t}}$ & $26.7 \mathrm{ab}$ & 1 & 2 & $0.48(0.39,0.58)$ & $0.30(0.12,0.48)$ & $\mathrm{SC}$ \\
\hline 39 & Pacemaker III & $27.1 \mathrm{ab}$ & 2 & 1 & $0.41(0.28,0.55)$ & $0.40(0.24,0.56)$ & $\mathrm{SC}$ \\
\hline 16 & Early Wonder Tall Top & $27.8 \mathrm{ab}$ & 1 & 2 & $0.46(0.35,0.58)$ & $0.34(0.18,0.51)$ & SC \\
\hline 24 & Chioggia Guardsmark & $28.5 \mathrm{ab}$ & 1 & 2 & $0.47(0.36,0.58)$ & $0.33(0.17,0.50)$ & $\mathrm{SC}$ \\
\hline 40 & Eagle & $28.5 \mathrm{ab}$ & 1 & 2 & $0.48(0.40,0.57)$ & $0.30(0.13,0.47)$ & $\mathrm{SC}$ \\
\hline 15 & Cylindra & $29.2 \mathrm{ab}$ & 2 & 1 & $0.43(0.30,0.55)$ & $0.38(0.22,0.55)$ & SS \\
\hline 18 & Zeppo & $29.6 \mathrm{ab}$ & 2 & 1 & $0.44(0.33,0.55)$ & $0.36(0.20,0.53)$ & SS \\
\hline 46 & Ruby Queen & $29.7 \mathrm{ab}$ & 2 & 1 & $0.47(0.40,0.54)$ & $0.31(0.13,0.48)$ & SS \\
\hline 37 & Vulture & $29.8 \mathrm{ab}$ & 1 & 2 & $0.46(0.33,0.58)$ & $0.36(0.19,0.52)$ & QP \\
\hline 34 & Boltardy & $30.1 \mathrm{ab}$ & 2 & 1 & $0.43(0.32,0.55)$ & $0.37(0.21,0.54)$ & QP \\
\hline 7 & MacGregor's Favorite & $31.8 \mathrm{ab}$ & 2 & 1 & $0.47(0.41,0.54)$ & $0.29(0.11,0.47)$ & SS \\
\hline 20 & Touchstone Gold & $32.0 \mathrm{ab}$ & 1 & 2 & $0.41(0.26,0.56)$ & $0.40(0.26,0.55)$ & QP \\
\hline 2 & Soldier & $32.0 \mathrm{ab}$ & 2 & 1 & $0.44(0.33,0.56)$ & $0.36(0.19,0.53)$ & QP \\
\hline 4 & Subeto & $32.6 \mathrm{ab}$ & 2 & 1 & $0.47(0.39,0.55)$ & $0.31(0.13,0.49)$ & SS \\
\hline 57 & $\mathrm{~W} 446 \mathrm{~B}^{\mathrm{s}}$ & $33.0 \mathrm{ab}$ & 2 & 3 & $0.46(0.33,0.60)$ & $0.30(0.00,0.60)$ & - \\
\hline 9 & Flat of Egypt & $33.5 \mathrm{ab}$ & 2 & 1 & $0.42(0.29,0.55)$ & $0.40(0.24,0.56)$ & QP \\
\hline 50 & Rhonda & $33.5 \mathrm{ab}$ & 2 & 1 & $0.46(0.36,0.55)$ & $0.33(0.16,0.51)$ & QP \\
\hline 43 & Burpee's Red Ball & $33.6 \mathrm{ab}$ & 1 & 2 & $0.46(0.34,0.57)$ & $0.35(0.20,0.51)$ & QP \\
\hline 38 & Solo & $33.7 \mathrm{ab}$ & 2 & 1 & $0.47(0.38,0.55)$ & $0.31(0.14,0.49)$ & QP \\
\hline 6 & Pablo & $34.0 \mathrm{ab}$ & 2 & 1 & $0.47(0.38,0.55)$ & $0.31(0.14,0.49)$ & SS \\
\hline 25 & Merlin & $34.1 \mathrm{ab}$ & 2 & 3 & $0.47(0.40,0.54)$ & $0.28(0.13,0.44)$ & QC \\
\hline 29 & Letherman's Green Top & $34.2 \mathrm{ab}$ & 2 & 1 & $0.44(0.31,0.56)$ & $0.37(0.21,0.54)$ & QP \\
\hline 8 & Lutz Green Leaf & $34.5 \mathrm{ab}$ & 1 & 2 & $0.45(0.32,0.57)$ & $0.36(0.21,0.52)$ & QP \\
\hline 14 & Taunus & $34.7 \mathrm{ab}$ & 2 & 3 & $0.47(0.40,0.54)$ & $0.28(0.12,0.44)$ & SS \\
\hline 44 & Albino & $35.1 \mathrm{ab}$ & 2 & 1 & $0.43(0.30,0.55)$ & $0.39(0.22,0.55)$ & QP \\
\hline 22 & Moneta & $35.6 \mathrm{ab}$ & 2 & 1 & $0.44(0.33,0.56)$ & $0.36(0.19,0.53)$ & QP \\
\hline 30 & Kestral & $37.0 \mathrm{ab}$ & 2 & 1 & $0.48(0.43,0.53)$ & $0.24(0.07,0.40)$ & QP \\
\hline 48 & Detroit Dark Red & $37.0 \mathrm{ab}$ & 2 & 1 & $0.44(0.33,0.56)$ & $0.36(0.20,0.53)$ & QP \\
\hline 13 & Detroit Supreme & $37.9 \mathrm{abc}$ & 2 & 1 & $0.47(0.40,0.54)$ & $0.30(0.12,0.47)$ & QP \\
\hline 35 & Crosby Egyptian & $38.8 \mathrm{abc}$ & 2 & 1 & $0.44(0.34,0.55)$ & $0.36(0.20,0.52)$ & QP \\
\hline 27 & White Detroit & $38.9 \mathrm{abc}$ & 2 & 1 & $0.47(0.39,0.55)$ & $0.31(0.13,0.48)$ & SS \\
\hline 54 & W448B & $39.3 \mathrm{abc}$ & 2 & 1 & $0.48(0.41,0.54)$ & $0.28(0.08,0.48)$ & QP \\
\hline 26 & First Crop & $39.3 \mathrm{abc}$ & 2 & 1 & $0.47(0.40,0.54)$ & $0.29(0.12,0.47)$ & QC \\
\hline 49 & Red Ace & $40.1 \mathrm{abc}$ & 2 & 3 & $0.48(0.42,0.53)$ & $0.26(0.10,0.42)$ & QP \\
\hline 36 & Paonazza D Egitto & $40.4 \mathrm{abc}$ & 2 & 3 & $0.46(0.37,0.55)$ & $0.31(0.15,0.46)$ & SS \\
\hline 23 & Avalanche & $41.3 \mathrm{abc}$ & 2 & 1 & $0.47(0.41,0.54)$ & $0.29(0.11,0.47)$ & QC \\
\hline 41 & Moulin Rouge & $42.1 \mathrm{abc}$ & 2 & 3 & $0.48(0.42,0.53)$ & $0.25(0.10,0.41)$ & QC \\
\hline 59 & W424B & $42.6 \mathrm{abc}$ & 2 & 3 & $0.47(0.40,0.54)$ & $0.29(0.13,0.45)$ & QP \\
\hline 42 & Burpee's Golden & $43.5 \mathrm{abc}$ & 2 & 1 & $0.46(0.36,0.55)$ & $0.33(0.16,0.51)$ & QC \\
\hline 32 & Blankoma & $49.0 \mathrm{abc}$ & 2 & 3 & $0.45(0.35,0.55)$ & $0.32(0.16,0.49)$ & QC \\
\hline 5 & Robin & $50.2 \mathrm{abc}$ & 2 & 3 & $0.46(0.36,0.55)$ & $0.32(0.16,0.47)$ & QC \\
\hline 55 & W411B & $51.2 \mathrm{abc}$ & 2 & 3 & $0.46(0.35,0.58)$ & $0.30(0.06,0.54)$ & QC \\
\hline 1 & Syngenta Golden & $51.9 \mathrm{bc}$ & 3 & 2 & $0.43(0.36,0.51)$ & $0.29(0.12,0.46)$ & SS \\
\hline 19 & Boldor & $53.2 \mathrm{bc}$ & 2 & 3 & $0.44(0.33,0.55)$ & $0.34(0.19,0.49)$ & QC \\
\hline 60 & $\mathrm{~W} 434 \mathrm{~B}^{\mathrm{r}}$ & $100.0 \mathrm{c}$ & 5 & - & $1.00(1.00,1.00)$ & - & - \\
\hline
\end{tabular}

${ }^{\mathrm{z}}$ Code refers to the accessions in the boxplots in Supplemental Fig. 3 and Supplemental Table 3.

$\mathrm{y}_{\mathrm{HSD}_{0.05}}=6.0$. Different letters indicate significant differences as determined by Tukey pairwise comparisons at $\alpha=0.05$.

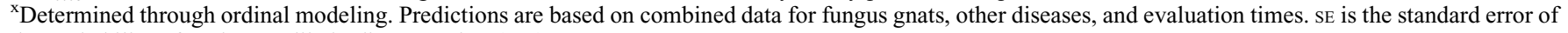
the probability of each most likely disease rating (DR).

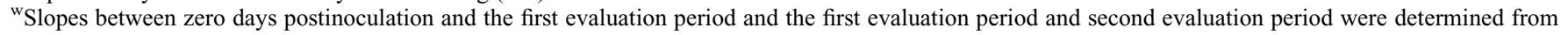

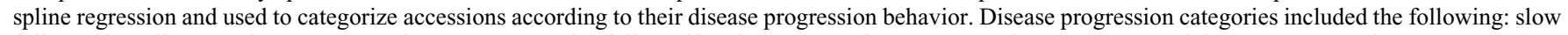

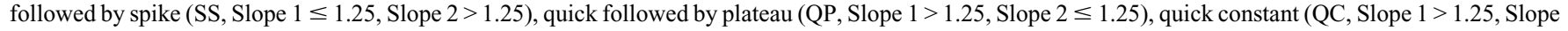
$2>1.25$ ), and slow constant (SC, Slope $1 \leq 1.25$, Slope $2 \leq 1.25$ ).

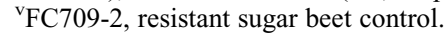

${ }^{\mathrm{u}} \mathrm{W} 364 \mathrm{~B}$, table beet inbred control.

${ }^{\mathrm{t}} \mathrm{FC} 901 / \mathrm{C} 817$, susceptible sugar beet control.

${ }^{\mathrm{s}}$ Disease progression category not provided as slopes could not be calculated, observations only at the second evaluation period.

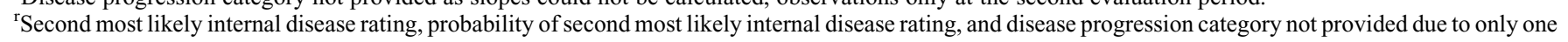
observation. 
Table 3. Mean diseased tissue percentage, most likely internal disease ratings and probabilities, and disease progression categories of 10 Beta vulgaris subsp. vulgaris commercial cultivars and publicly available accessions in response to inoculation with Rhizoctonia solani AG 2-2 isolate R1 in a greenhouse screen evaluated in 2019 .

\begin{tabular}{|c|c|c|c|c|c|c|c|}
\hline $\operatorname{Code}^{z}$ & Accession & $\begin{array}{l}\text { Mean diseased } \\
\text { tissue percentage }\end{array}$ & $\begin{array}{l}\text { First most likely } \\
\text { internal } \mathrm{DR}^{\mathrm{x}}\end{array}$ & $\begin{array}{l}\text { Second most likely } \\
\text { internal } \mathrm{DR}^{\mathrm{x}}\end{array}$ & 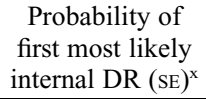 & $\begin{array}{c}\text { Probability of } \\
\text { second most likely } \\
\text { internal DR (SE) }\end{array}$ & Disease progression $^{\mathrm{w}}$ \\
\hline 6 & Badger Flame & $29.4 \mathrm{a}$ & 2 & 1 & $0.37(0.31,0.42)$ & $0.25(0.18,0.31)$ & $\mathrm{SC}$ \\
\hline 1 & FC709-2 ${ }^{v}$ & $31.8 \mathrm{ab}$ & 2 & 1 & $0.37(0.31,0.42)$ & $0.25(0.19,0.32)$ & $\mathrm{SC}$ \\
\hline 10 & Detroit Supreme & $36.9 \mathrm{abc}$ & 2 & 3 & $0.37(0.32,0.43)$ & $0.26(0.17,0.35)$ & QP \\
\hline 7 & Moneta & $39.1 \mathrm{abc}$ & 2 & 3 & $0.37(0.32,0.43)$ & $0.28(0.19,0.38)$ & QP \\
\hline 8 & Soldier & $42.2 \mathrm{bc}$ & 2 & 3 & $0.37(0.32,0.43)$ & $0.29(0.20,0.39)$ & QC \\
\hline 4 & W364B ${ }^{\mathrm{u}}$ & $42.8 \mathrm{~cd}$ & 2 & 1 & $0.35(0.29,0.42)$ & $0.27(0.21,0.34)$ & QP \\
\hline 2 & $\mathrm{FC} 901 / \mathrm{C} 817^{\mathrm{t}}$ & $45.6 \mathrm{~cd}$ & 2 & 3 & $0.37(0.31,0.42)$ & $0.25(0.16,0.33)$ & $\mathrm{QC}$ \\
\hline 9 & Touchstone Gold & $47.5 \mathrm{~cd}$ & 2 & 3 & $0.37(0.32,0.42)$ & $0.34(0.24,0.45)$ & QP \\
\hline 3 & KDH13 & $53.5 \mathrm{~d}$ & 3 & 2 & $0.42(0.31,0.52)$ & $0.35(0.30,0.41)$ & QP \\
\hline$\underline{5}$ & W411B & $54.9 \mathrm{~d}$ & 3 & 2 & $0.49(0.36,0.61)$ & $0.31(0.23,0.40)$ & $\mathrm{QC}$ \\
\hline
\end{tabular}

${ }^{\mathrm{z}}$ Code refers to the accessions in Supplemental Fig. 1 and Supplemental Table 1.

${ }_{\mathrm{HSD}_{0.05}}=4.5$. Different letters indicate significant differences as determined by Tukey pairwise comparisons at $\alpha=0.05$.

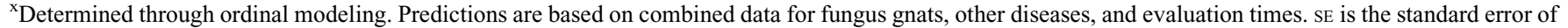
the probability of each most likely disease rating (DR).

${ }^{\text {w}}$ Slopes between zero days postinoculation and the first evaluation period and the first evaluation period and second evaluation period were determined from spline regression and used to categorize accessions according to their disease progression behavior. Disease progression categories included the following: slow followed by spike (SS, Slope $1 \leq 1.25$, Slope $2>1.25$ ), quick followed by plateau (QP, Slope $1>1.25$, Slope $2 \leq 1.25)$, quick constant $(\mathrm{QC}$, Slope $1>1.25$, Slope $2>1.25$ ), and slow constant (SC, Slope $1 \leq 1.25$, Slope $2 \leq 1.25$ ).

${ }^{v}$ FC709-2, resistant sugar beet control.

${ }^{\mathrm{u}} \mathrm{W} 364 \mathrm{~B}$, table beet inbred control

${ }^{\mathrm{t}} \mathrm{FC} 901 / \mathrm{C} 817$, susceptible sugar beet control.

honestly significant difference test (at $\alpha=$ 0.05 ) in "agricolae" was used to separate means (de Mendiburu, 2019). The Wilcoxon rank sum test for independent samples was used to validate the results of the PI and Commercial Screens. The weighted average diseased tissue percentages of the accessions in the Validation Screen were compared with their counterparts in the Commercial and PI Screens.

In spline regression models, AUDPC was included as the dependent variable to determine the slopes between zero days postinoculation to the first evaluation time and between the first and second evaluation periods. These slopes were then used to assign accessions to the following disease progression categories: slow followed by spike (Slope $1 \leq 1.25$, Slope $2>1.25$ ), quick followed by plateau (Slope $1>1.25$, Slope $2 \leq 1.25$ ), quick constant (Slope $1>1.25$, Slope $2>1.25$ ), and slow constant (Slope $1 \leq$ 1.25 , Slope $2 \leq 1.25$ ).

Linear regression has limitations when analyzing ordinal data such as those collected in these experiments. Liddell and Kruschke (2018) demonstrated that when ordinal variables are analyzed as metric, low rates of correct detection, inflated type I error rates, inversions of differences between groups, and distorted effect-size estimates can occur. Therefore, in addition to linear regression, we also conducted ordinal regression to analyze all data and predict the most likely disease rating for each accession. We used the "ordinal" package in $\mathrm{R}$ to fit ordinal models (Christensen, 2019) and a "cumulative" ordinal model as described by Bürkner and Vuorre (2019). Internal and external disease ratings were modeled separately to maintain the integrity of each rating. Because internal characteristics are of greater importance in the vegetable processing industry, we focus on those in our presentation of the results. Because of constraints of ordinal modeling, subsamples were not averaged.

\section{Results}

Validation screen. Significant interactions were found for replicate (i.e., experimental run) with evaluation time, other diseases, and inoculation $(P<0.001,0.05$, and 0.05 , respectively). However, replicate $\times$ accession interactions were not significant $(P>0.05)$. Therefore, replicate was excluded from the model and both replicates were combined for analysis. ANOVA showed main effects of accession, fungus gnats, other diseases, and inoculation to be highly significant for average weighted diseased tissue (Table 4).

Accession $\times$ other disease and accession $\times$ inoculation interactions were significant $(P<$ 0.01 and 0.001 , respectively).

Most inoculated plants exhibited diseased tissue ranging from $20 \%$ to $60 \%$ (Table 3, Supplemental Fig. 1) compared with the noninoculated checks in the Validation Screen. Noninoculated checks showed no to very little diseased tissue (less than $2 \%$ ). The percentage of diseased tissue was higher in the 5-week postinoculation evaluation time period compared with the 3 -week time period.

Ordinal regression predicted that, in the absence of fungus gnats and other diseases, many accessions were likely to have an internal disease rating of 1 or 2 (Table 3 ). In contrast, when fungus gnats and other diseases were present, these accessions were predicted to have an internal disease rating of a 2 or 3 (data not shown).

Ordinal models predicted the likelihood of accessions receiving a specific internal disease rating (Table 3). 'Badger Flame' and FC709-2 were the most resistant in the screen with mean diseased tissue percentages of 29 and 32, respectively. KDH13 and W411B were on the susceptible end, with mean diseased tissue percentages of 54 and 55 , respectively. It is interesting to note that both 'Badger Flame' and FC709-2 were most likely to receive a 2 internal disease rating (both $P=0.37$ ), followed by a 1 internal disease rating (both $P=0.25$ ). On the other end, KDH13 and W411B were most likely to receive a 3 internal disease rating $(P=0.42$ and 0.49 , respectively), followed by a 2 internal disease rating $(P=0.35$ and 0.31 , respectively). Both of the most resistant accessions also demonstrated a disease progression that was slow constant (Table 3, Supplemental Fig. 1, Supplemental Table 1). The other eight accessions in this screen exhibited either a quick constant or quick followed by plateau disease progression.

PI screen. Replicate $\times$ accession interaction was not significant $(P=0.95)$, and therefore replicate was excluded from the model and replicates were combined for analysis. ANOVA demonstrated significant main effects contributing to the percentage of diseased tissue, including accession, fungus gnats, other diseases, evaluation time, and inoculation (Table 5).

In general, inoculated plants exhibited between $6 \%$ and $40 \%$ diseased tissue (Table 1, Supplemental Fig. 2) compared with no or very little diseased tissue (an average of $2 \%$ ) in the noninoculated checks. The accessions in the PI Screen exhibited increased amounts of diseased tissue in the 5 -week postinoculation evaluation period compared with the 3 -week evaluation period.

Ordinal models predicted the likelihood of accessions receiving a specific internal disease rating (Table 1). Ames 22164 and PI 502294 were the most resistant in the 


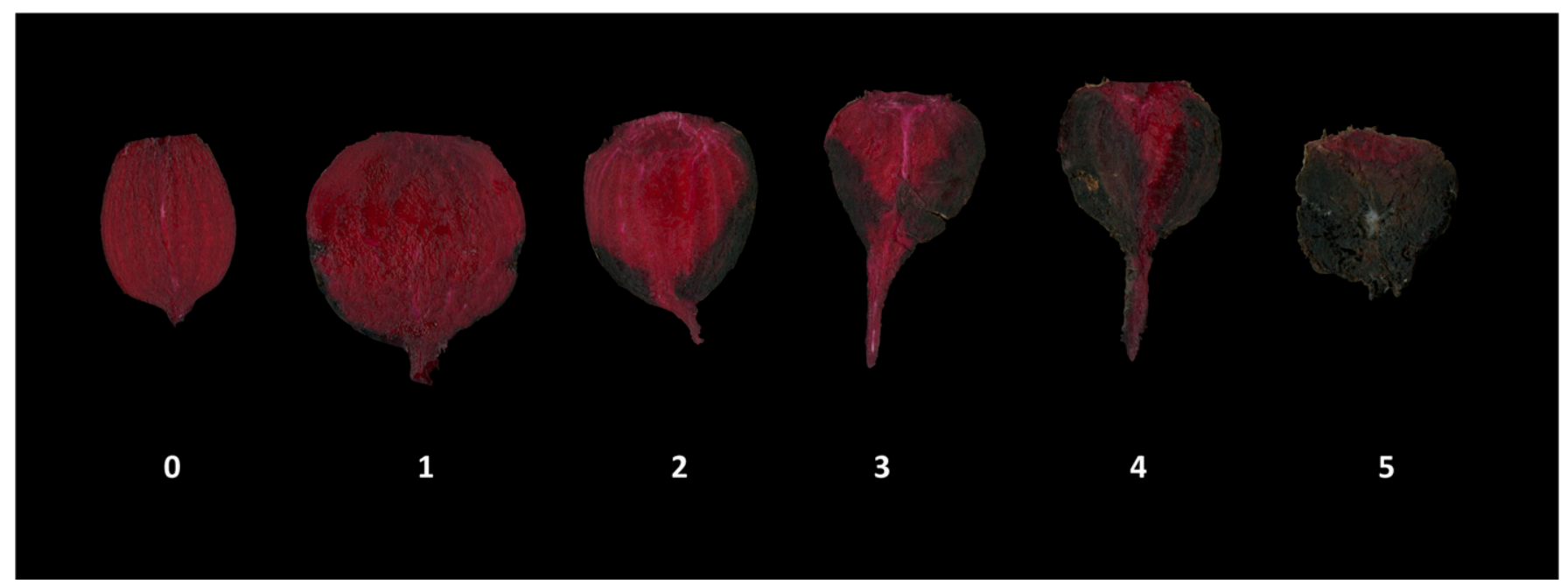

Fig. 1. Disease rating scale for Beta vulgaris subsp. vulgaris inoculated with Rhizoctonia solani AG 2-2 isolate R1. $0=0 \%$ diseased tissue, root surface clean with no visible lesions; $1=1 \%$ to $10 \%$ diseased tissue, superficial, scattered nonactive lesions; $2=11 \%$ to $30 \%$ of root affected; $3=31 \%$ to $60 \%$ of root affected; $4=$ $61 \%$ to $99 \%$ of root blackened with rot extending into interior; and $5=$ root $100 \%$ rotted and foliage is dead or dying.

Table 4. Analysis of variance for the average weighted diseased tissue percentage of 10 Beta vulgaris subsp. vulgaris commercial cultivars and publicly available accessions in response to inoculation with Rhizoctonia solani AG 2-2 isolate R1 in a greenhouse screen evaluated in 2019.

\begin{tabular}{lrrr}
\hline Source & df & Mean squares & Significance \\
\hline Accession & 9 & 1,312 & $* * *$ \\
Fungus gnats & 1 & 17,799 & $* * *$ \\
Other diseases & 1 & 3,035 & $* * *$ \\
Evaluation time & 1 & 127 & Ns \\
Inoculation & 1 & 11,080 & Ns \\
Accession: fungus gnats & 9 & 24 & $* *$ \\
Accession: other diseases & 8 & 316 & $* * *$ \\
Accession: inoculation & 2 & 1,438 & Ns \\
Fungus gnats: other diseases & 1 & 340 & NS \\
Accession: fungus gnats: other diseases & 4 & 122 & 103 \\
Residuals & 169 & &
\end{tabular}

NS $, * *, * * *$ Nonsignificant or significant at $P \leq 0.01$ or 0.001 , respectively.

screen with mean diseased tissue percentages of 2 and 6, respectively. PI 590595 and PI 103042 were on the susceptible end, both with mean diseased tissue percentages of 44 . Ames 22164 and PI 502294 were most likely to receive a 0 and 1 internal disease rating, respectively $(P=0.56$ and 0.47 , respectively), followed by a 1 and 2 internal disease rating $(P=0.37$ and 0.30 , respectively). On the other end, PI 590595 and PI 103042 were most likely to receive a 2 internal disease rating $(P=0.44$ and 0.47 , respectively), followed by an internal disease rating of 3 and 1 , respectively $(P=0.28$ and 0.29 , respectively).

Predictions from ordinal modeling demonstrated the effect of fungus gnats and other diseases on the response of beets to inoculation with $R$. solani. In the presence of fungus gnats and other diseases, most accessions in the PI Screen were predicted to have an internal disease rating of 1 (Table 1). In contrast, when fungus gnats and other diseases were present, most accessions were predicted to have an internal disease rating of 2 (data not shown). Interactions between accession and fungus gnats and accession and inoculation were significant as well.
Many of the most resistant accessions in the PI Screen had mean diseased tissue percentages less than that of the resistant sugar beet check (Table 1, Supplemental Fig. 2). The 11 most resistant accessions also demonstrated a disease progression that was slow constant (Supplemental Fig. 2, Supplemental Table 2). Of 97 accessions, most at 67 had disease progressions that were slow constant, 28 were slow followed by spikes, and only two were quick followed by plateaus.

Commercial screen. Replicate $\times$ accession interactions were not significant $(P=$ $0.24)$. We attributed replicate significance to the replicate $\times$ fungus gnat interaction $(P<$ $0.01)$. Therefore, replicate was excluded from the model and both replicates were combined for analysis. ANOVA showed all main effects to be highly significant $(P<$ 0.01 ) for average weighted diseased tissue (Table 6).

Most inoculated plants exhibited diseased tissue ranging from $16 \%$ to $50 \%$ (Table 2 , Supplemental Fig. 3) compared with the noninoculated checks (an average of less than $1 \%$ diseased tissue) in the Commercial Screen. The percentage of diseased tissue was higher in the 5-week postinoculation evaluation time period compared with the 3-week time period.

Ordinal models predicted the likelihood of accessions receiving a specific internal disease rating (Table 2). FC709-2 and Long Season were the most resistant in the screen with mean diseased tissue percentages of 7 and 16, respectively. Syngenta Golden, Boldor, and W434B were on the susceptible end, with mean diseased tissue percentages of 52 , 53, and 100.0, respectively. Both FC709-2 and Long Season were most likely to receive a 1 internal disease rating $(P=0.49$ and 0.48 , respectively), followed by an internal disease rating of 0 and 2 , respectively $(P=0.31$ and 0.32 , respectively). On the other end, Syngenta Golden, Boldor, and W434B were most likely to receive a 3,2 , and 5 internal disease rating, respectively $(P=0.43,0.44,1.00$, respectively). The second most likely internal disease rating for Syngenta Golden and Boldor was a 2 and 3 , respectively $(P=$ 0.29 and 0.34 , respectively).

Ordinal regression predicted that, in the absence of fungus gnats and other diseases, many accessions were likely to have an internal disease rating of 1 (Table 2). In contrast, when fungus gnats and other diseases were present, these accessions were predicted to have an internal disease rating of a 2 or 3 (data not shown).

The most resistant accessions in the Commercial Screen had mean diseased tissue percentages similar to that of FC709-2 (Table 2, Supplemental Fig. 3). These resistant accessions also demonstrated a disease progression that was slow constant (Supplemental Fig. 3, Supplemental Table 3). Of 62 accessions, 17 had disease progressions that were slow constant, 12 slow followed by spikes, 22 quick followed by plateaus, and 9 quick constant.

Variance of mean diseased tissue percentage was $223.4,263.9$, and 300.4 across accessions for percent diseased tissue in the Validation, PI, and Commercial Screens, 
Table 5. Analysis of variance for the average weighted diseased tissue percentage of 97 Beta vulgaris subsp. vulgaris PI accessions in response to inoculation with Rhizoctonia solani AG 2-2 isolate R1 in a greenhouse screen evaluated in 2018.

\begin{tabular}{lrrr}
\hline Source & df & Mean squares & Significance \\
\hline Accession & 96 & 254 & $* * *$ \\
Fungus gnats & 1 & 39,659 & $* * *$ \\
Other diseases & 1 & 4,817 & $* *$ \\
Evaluation time & 1 & 1,004 & $* * *$ \\
Inoculation & 1 & 1,622 & $* *$ \\
Accession: fungus gnats & 83 & 156 & NS \\
Accession: other diseases & 59 & 105 & $* *$ \\
Accession: inoculation & 2 & 579 & $*$ \\
Fungus gnats: other diseases & 1 & 543 & Ns \\
Accession: fungus gnats: other diseases & 1 & 71 & 93 \\
Residuals & 153 & & \\
\hline
\end{tabular}

NS $, *, * *, * * *$ Nonsignificant or significant at $P \leq 0.05,0.01$, or 0.001 , respectively.

Table 6. Analysis of variance for the average weighted diseased tissue percentage of 62 Beta vulgaris subsp. vulgaris commercial cultivars and publicly available inbred accessions in response to inoculation with Rhizoctonia solani AG 2-2 isolate R1 in a greenhouse screen evaluated in 2018.

\begin{tabular}{lrrr}
\hline Source & df & Mean squares & Significance \\
\hline Accession & 61 & 534 & $* * *$ \\
Fungus gnats & 1 & 13,865 & $* * *$ \\
Other diseases & 1 & 2,882 & $* *$ \\
Evaluation time & 1 & 1,557 & $* *$ \\
Inoculation & 1 & 1,647 & NS \\
Accession: fungus gnats & 51 & 190 & NS \\
Accession: other diseases & 31 & 196 & NS \\
Accession: inoculation & 2 & 113 & NS \\
Fungus gnats: other diseases & 1 & 32 & 162 \\
Residuals & 104 & &
\end{tabular}

NS $* *, * * *$ Nonsignificant or significant at $P \leq 0.01$ or 0.001 , respectively.

respectively, demonstrating consistency in the three screening experiments.

\section{Discussion}

The methods described for a controlled environment screen for $R$. solani in table beet provides a valuable assessment tool for table beet breeding programs. In our greenhouse experiments, we were able to create a screening environment that was repeatable and consistent. Using increased replications of accessions in the Validation Screen, we validated our findings from the PI and Commercial Screens. Even though each screen had a different mean diseased tissue percentage and range of values, the Validation Screen generally confirmed the relative performance of the two sugar beet control genotypes and a table beet inbred that were included in all three screens. Our screen was adapted from those conducted with sugar beet (Hecker and Ruppel, 1977b; L. Panella, personal communication; Ruppel et al., 1979). Many studies have demonstrated consistency between laboratory or greenhouse and field settings for sugar beet (Büttner et al., 2004; Campbell and Altman, 1976; Schneider et al., 1982; Scholten et al., 2001; Weiland et al., 1999), therefore, there is a high likelihood that our findings will be applicable in field environments for table beet.

We identified substantial variation in diseased tissue among all three types of accessions we evaluated, demonstrating a range of reaction to inoculation with $R$. solani AG 2-2 in table beet. These results are not particularly surprising given that there has been little direct selection in table beet for resistance to $R$. solani. The two sugar beet accessions we included as controls performed comparably to the same or similar accessions in screens completed in other sugar beet breeding programs (Hanson, 2010; Panella, 1999; Strausbaugh et al., 2013a). In our and other screens, FC709-2 had less diseased tissue compared with FC901/C817 (Panella, 1999; Panella and Hanson, 2001; Strausbaugh et al., 2013a). We found some table beet accessions that performed as well as the resistant sugar beet check genotype, suggesting promise for breeding in table beet. Some of the top performing accessions included 'Badger Flame', 'Long Season', 'Red Cloud', 'Albina Vereduna', and numerous PIs. Many of these accessions had AUDPCs similar to or less than that of that of the resistant sugar beet check.

Many of the more resistant accessions in our experiments displayed a disease progression that was slow constant (Tables 1-3, Supplemental Figs. 1-3, Supplemental Tables 1-3). Accessions with higher mean diseased tissue percentages exhibited a disease progression that was slow followed by spike, quick followed by plateau, or quick constant. In the Commercial Screen especially, most of the accessions at the susceptible end displayed a quick constant disease progression. Although some variability may occur between the first and second evaluation periods due to individual plant differences as well as variability in response over time, the disease progression categories obtained from the slopes of the AUDPC curve may be useful for informing selection programs aimed at improving resistance. Selection for the slow constant disease progression type may result in germplasm with greater resistance to $R$. solani. Planting of accessions with a slow constant disease progression may also provide more flexibility for harvest windows for growers.

We were also interested in determining the best method of measuring diseased tissue percentages using both external and internal observations. Using the ratio of 75 internal:25 external diseased tissue percentages, we found that most accessions ranged between a disease rating of 1 and 3 . Using this weighted average, variances for percent diseased tissue were relatively consistent across the screens with variances of 223.4, 263.9, and 300.4 for mean disease tissue percentage in the Validation, PI, and Commercial Screens, respectively. However, had we focused on strictly external diseased tissue, most accessions would have ranged between a disease rating of 1 and 5 and we would have found variances of 444.3, 856.6, and 722.9 for mean disease tissue percentage across accessions in the Validation, PI, and Commercial Screens, respectively. If we had only considered internal diseased tissue, most accessions would have received a disease rating between 0 and 3 and mean disease tissue percentage variances were tighter at 236.8 , 194.1, and 277.2 for the Validation, PI, and Commercial Screens, respectively. This finding indicates that there was more variation in the external root symptoms compared with their internal symptoms. Other studies have observed variation in plant parts in response to inoculation with $R$. solani AG 2-2 (Scholten et al., 2001). In greenhouse studies, Scholten et al. (2001) observed that leaf symptoms in sugar beet plants were widely variable compared with root symptoms and not correlated. In addition, rank changes among accessions occurred when either internal or external ratings were considered separately. This is because some accessions, like 'Eagle', had a large amount of diseased tissue on the root surface and very little diseased tissue internally. Other accessions, like 'Red Ace', had more extensive internal disease, with less externally. We focused on the internal symptoms in our studies because that is of greater importance in the table beet processing industry. In sugar beet experiments with $R$. solani, disease evaluations are typically done using root surface area (Ruppel et al., 1979; Scholten et al., 2001). Gaining a better understanding of the relationship between external and internal characteristics may allow for increased efficiency in disease evaluations. Further, the differences in internal and external variances suggest that genetic variation for external and internal disease reaction may be inherited separately in table beet.

We had expected the PI accessions to have the greatest amount of variation given that germplasm bank accessions typically exhibit large amounts of variation for performance traits (Frese et al., 2001; Panella and Lewellen, 2007). For example, of the 803 B. vulgaris subsp. vulgaris accessions classified 
as sugar beet type previously screened for $R$. solani AG 2-2, more than 50 accessions were rated at a 3 or lower on a 0 to 9 or very resistant to highly susceptible disease rating scale (GRIN, 2020). Sugar beet cyst nematode screens of NPGS Beta germplasm have shown that a number of accessions were segregating for resistance to the nematode (Panella and Lewellen, 2007). Prebreeding populations of sugar beet derived from PIs also demonstrated segregation for reaction to the destructive oomycete, Aphanomyces cochlioides Dresch (Panella and Lewellen, 2007). Surprisingly, we observed larger variation among commercial cultivars than among PI accessions. This might be because some of the accessions in the Commercial Screen are open-pollinated (OP) or heirloom cultivars, whereas others are $F_{1}$ hybrids. Some examples of each of these are 'Soldier' and 'Ruby Queen' (OP/heirloom) and 'Red Ace' and 'Boldor' (hybrids) with variances in diseased tissue percentage of 106.6, 366.9, 271.8, and 472.6, respectively. Hybrids generally exhibited greater variation than heirloom and OP cultivars, whereas inbred lines exhibited the lowest amount of variation.

Some of the most resistant accessions in the PI Screen were commercial cultivars that had been contributed to the NPGS, but there were many other resistant accessions that were not cultivars. This provides additional evidence for the utility of the table beet germplasm in the NPGS for increasing resistance to $R$. solani AG 2-2 in table beet lines. However, the accessions in the Commercial Screen displayed a smaller increase in mean diseased tissue percentage from the 3- to 5week postinoculation evaluation periods compared with a greater increase in mean diseased tissue percentage in the PI Screen. This finding indicates that many commercial accessions may be better able to resist the progression of disease, and may be an important consideration in breeding for disease resistance and disease management from a grower perspective.

The predictions provided by ordinal regression can be used to make better informed decisions when selecting germplasm for breeding and selecting varieties for production. Predictions can be used to estimate how much diseased tissue can be expected on an accession when $R$. solani is present. We observed significant interactions between accession $\times$ fungus gnats, accession $\times$ other diseases, and accession $\times$ inoculation. It is expected that accessions with different genetic backgrounds will respond differently to environmental factors. The role of environment and microclimate surrounding individual plants in the development of Rhizoctonia root and crown rot on sugar beet has been noted by Hecker and Ruppel (1977b). Others have examined the effects of temperature and moisture on R. solani (Bolton et al., 2010; Engelkes and Windels, 1994; Kirk et al., 2008; McKeen, 1949). Bolton et al. (2010) observed a positive correlation between root disease index and increased temperature and soil moisture.
We noted that when fungus gnats and other diseases were present, accessions were more likely to exhibit an increased percentage of diseased tissue. Such relationships or disease complexes for $R$. solani have been described with Fusarium oxysporum f. sp. radis-betae (Harveson and Rush, 1994; Jacobsen, 2006), Pythium spp. (Abawi et al., 1986), and A. cochlioides (Harveson and Rush, 1994; Harveson et al., 2002). In Michigan, sugar beets with resistance to $R$. solani were found to have more severe root rot when inoculated with both $R$. solani and Rhizopus stolonifer (Ehrenberg) Vuillemin than either fungus by itself (Hanson, 2010). This is especially noteworthy, because the growing conditions in this study were not conducive to disease production by $R$. stolonifer on its own. The relationship between $R$. solani and bacteria in root rot complexes was investigated by Strausbaugh and Gillen (2009). In field studies, fungal and bacterial rot only developed on plants inoculated with $R$. solani in the crown area, when no fungal infection was present, neither was bacterial rot (Strausbaugh and Gillen, 2009). Later experiments showed a doubled amount of root rot when both the bacteria Leuconostoc mesenteroides subsp. dextranicum (Beijerinck) Garvie and $R$. solani AG 2-2 IIIB were inoculated on sugar beets compared with $R$. solani alone and no rot when $L$. mesenteroides was inoculated alone, further demonstrating synergism between the bacteria and fungus (Strausbaugh et al., 2013b).

Interactions between fungi and insects may also be a factor in the response to $R$. solani and Hatcher (1995) provides an extensive review of relationships between pathogenic fungi, insects, and host plants. Increased levels of root damage and decreased alfalfa yield occurred when both clover root curculio, Sitona hispidulus (Fabricius), and a complex of soilborne root rot fungi were present compared with either pest alone (Godfrey and Yeargan, 1987). Caesar (2003) observed a synergistic relationship when $R$. solani and $F$. oxysporum were inoculated in combination with Aphthona spp., flea beetle, leading to increased damage on the roots on Euphorbia esulalvirgata. These synergistic relationships between $R$. solani and other plant pathogenic organisms are of great importance for management practices and breeding. There is a need for the development of beet lines with resistance to multiple diseases (Gaskill et al., 1970; Harveson and Rush, 1994; Jacobsen et al., 2004). Incorporating multiple disease resistances will be time-consuming, as resistance to several of the most destructive pathogens, including Rhizoctonia (Afanasiev and Sharp, 1961; Hecker and Ruppel, 1975) and Aphanomyces (Afanasiev and Sharp, 1961) is polygenic.

These experiments provide a good foundation for the study of resistance to $R$. solani in table beet. However, there are many areas that can be explored further to gain a better understanding of the nature of resistance in table beet, one of which is exploring possible interactions between $R$. solani AG 2-2 isolates and table beet genotype. In our experiments, we used isolate R1 to inoculate the beets, because it is the isolate of choice for other sugar beet disease research programs (L. Hanson, personal communication). In early experiments, Herr (1970) inoculated eight sugar beet breeding lines developed by the USDA-ARS Crops Research Laboratory with three highly virulent Ohio $R$. solani isolates and discovered that the breeding lines were also resistant to Ohio isolates. Other experiments demonstrated no interactions between $R$. solani isolates from sugar beet roots and sugar beet lines (Ruppel, 1972). Hecker and Ruppel (1977b) tested six $R$. solani isolates on both resistant and susceptible sugar beets and found no significant interaction between fungal strain and sugar beet genotype. Hecker and Ruppel (1977b) also discussed experiments done by Sugimoto in Hokkaido, Japan, that confirmed similar levels of resistance exhibited by FC $701 / 2$ and FC702/2 inoculated with two Hokkaido $R$. solani isolates. These two breeding lines exhibited the highest level of resistance in Sugimoto's field and greenhouse experiments (Hecker and Ruppel, 1977b). The applicability of resistance to Colorado isolates was again demonstrated by Windels et al. (1995) when six resistant lines were inoculated with Colorado $R$. solani AG 2-2 IIIB isolate R9 and four highly virulent Minnesota isolates in field experiments in both Colorado and Minnesota (Strausbaugh et al., 2011). However, in the 2000 s some of these resistant sugar beet cultivars were not performing as expected in the U.S. Intermountain West (IMW) region. Greenhouse pathogenicity tests with 94 IMW region isolates on a susceptible sugar beet line led Strausbaugh et al. (2009) to recommend more study was needed for the isolates used for host resistance selection. On further investigation, Strausbaugh et al. (2013a) determined that although interactions with strains of $R$. solani AG 2-2 IIIB were not present with the susceptible and highly resistant checks (FC901/C817 and FC 709-2, respectively), sugar beet lines with intermediate levels of resistance had significant interactions with the nine isolates tested. This supports the necessity of carefully selecting isolates for screening in disease breeding nurseries. It would be interesting to compare how different isolates of $R$. solani AG 2-2IIIB affect table beets. Collecting isolates from local fields would allow researchers to screen germplasm against the isolates most specific to their geographic region, allowing for local adaptation of germplasm.

To screen germplasm with $R$. solani on table beet plants while containing the pathogen, we conducted our experiments in greenhouses. Despite being in a controlled environment and making applications of $B$. thuringiensis ssp. israelensis, fungus gnats and symptoms atypical of $R$. solani, including brown and gray tissue and vascular discoloration, were present on the specimens. 
Although other diseases are likely to be present in a field environment, their presence in our experiments made it more difficult to precisely measure the performance of germplasm in response to the fungus. Several studies have been conducted by inoculating seeds and seedlings in sugar beet (Bolton et al., 2010; Liu et al., 2019; Nagendran et al., 2009). In his discussion of breeding for resistance to Rhizoctonia in sugar beet, Gaskill (1968) mentions that resistance in mature sugar beets developed through selection was ineffective at preventing losses to Rhizoctonia in seedlings. This was later supported by Bolton et al. (2010) when several AGs and ISGs were capable of causing disease on sugar beet seedlings despite host resistance. A recent study by Liu et al. (2019) examined commercial cultivars that were susceptible, moderately resistant, and resistant to $R$. solani and found that no significant resistance to AG 2-2 IIIB was exhibited by the resistant cultivars until 4 and 5 weeks of age. In a study investigating host-pathogen interactions of $R$. solani and sugar beet seedlings, EL51, a line with resistance to Rhizoctonia crown and root rot, was discovered to have damping off resistance to AG 2-2 IIIB when inoculated 2 weeks after germination (Halloin et al., 2000; Nagendran et al., 2009). Another approach might be to directly inoculate portions of table beet roots in a laboratory environment. In studies of plant pathogens affecting leaves, such experiments are referred to as "detached leaf assays" (Nowakowska et al., 2014). Such procedures have also been referred to as "direct challenge assays" (Woodward and Pearce, 1988) and may allow for evaluation of more precise responses to $R$. solani. The results presented here demonstrate a 13-week greenhouse screening method to separate genotypes in their response to inoculation by $R$. solani, identify useful germplasm, and make progress toward breeding for host resistance in table beet.

\section{Literature Cited}

Abawi, G.S., D.C. Crosier, A.C. Cobb, and R.F. Becker. 1986. Root rot of table beets in New York State. N.Y. Food Life Sci. Bul. 115. $<$ http://vegetablemdonline.ppath.cornell.edu/>.

Abawi, G.S., J.R. Kikkert, K. Moktan, H.W. Lange, and C.D Smart. 2014. First report of resistance to Quadris among populations of Cercospora beticola causing leaf spot of table beets in New York State. Phytopathology 104(Suppl. 1):S1.1 (abstr.).

Afanasiev, M.M. and E.L. Sharp. 1961. Testing of inbred lines of sugar beets for resistance to Aphanomyces, Rhizoctonia and Fusarium root rots. J. Amer. Soc. Sugar Beet Technol. 11:542-546.

Arabiat, S. and M.F.R. Khan. 2016. Sensitivity of Rhizoctonia solani AG-2-2 from sugar beet to fungicides. Plant Dis. 100:2427-2433.

Bolton, M.D., L. Panella, L. Campbell, and M.F.R. Khan. 2010. Temperature, moisture, and fungicide effects in managing Rhizoctonia root and crown rot of sugar beet. Phytopathology 100:689-697.

Bürkner, P.C. and M. Vuorre. 2019. Ordinal regression models in psychology: A tutorial. Adv. Methods Pract. Psychol. Sci. 2:77-101.
Büttner, G., B. Pfähler, and B. Märländer. 2004. Greenhouse and field techniques for testing sugar beet for resistance to Rhizoctonia root and crown rot. Plant Breed. 123:158-166.

Büttner, G., M.F. Ithurrart, and J. Buddemeyer. 2002. Root and crown rot Rhizoctonia solani: Distribution, economic importance and concepts of integrated control (in German). Zuckerindustrie 127:856-866.

Caesar, A.J. 2003. Synergistic interaction of soilborne plant pathogens and root-attacking insects in classical biological control of an exotic rangeland weed. Biol. Control 28:144-153.

Campbell, C.L. and J. Altman. 1976. Rapid laboratory screening of sugar beet cultivars for resistance to Rhizoctonia solani. Phytopathology 66:1373-1374.

Campbell, L.G., C.E. Windels, K.K. Fugate, and J.R. Brantner. 2014. Postharvest losses associated with severity of Rhizoctonia crown and root rot of sugarbeet at harvest. J. Sugar Beet Res. 51:31-51.

Cattanach, A.W., A.G. Dexter, and E.S. Oplinger. 1991. Sugarbeet. University of WisconsinMadison Extension. 29 May 2020. <http:// corn.agronomy.wisc.edu/>.

Christensen, R.H.B. 2019. Ordinal - Regression Models for Ordinal Data. R package version 2019.4-25. <http://www.cran.r-project.org/>.

Cloyd, R.A. 2010. Fungus gnat management in greenhouses and nurseries. Kansas State Univ. Agr. Expt. Sta. Coop. Ext. Serv. MF-2937. $<$ https://bookstore.ksre.ksu.edu/>.

Colquhoun, J.B., S.A. Chapman, A.J. Gevens, R.L. Groves, D.J. Heider, B.M. Jensen, G.R.W. Nice, M.D. Ruark, and Y. Wang. 2020. Table beet, p. 295-300. In: Commercial vegetable production in Wisconsin-2020 A3422. Univ. Wisconsin-Madison Ext. <https://learningstore. extension.wisc.edu/>.

Cubeta, M.A. and R. Vilgalys. 1997. Population biology of the Rhizoctonia solani complex. Phytopathology 87:480-484.

de Mendiburu, F. 2019. Agricolae: Statistical procedures for agricultural research. 15 Aug. 2019. $<\mathrm{http}$ ://www.cran.r-project.org/>.

Engelkes, C.A. and C.E. Windels. 1994. Relationship of plant age, cultivar, and isolate of $R h i-$ zoctonia solani AG-2-2 to sugar beet root and crown rot. Plant Dis. 78:685-689.

Engelkes, C.A. and C.E. Windels. 1996. Susceptibility of sugar beet and beans to Rhizoctonia solani AG2-2 IIIB and AG-2-2 IV. Plant Dis. 80:1413-1417.

Eujayl, I.A., C.A. Strausbaugh, and L. Chunsheng. 2016. Registration of sugar beet doubled haploid line KDH13 with resistance to beet curly top. J. Plant Regist. 10:93-96.

Fenwick, A.L., T. Vagher, A. Nielson, and L. Panella. 2018. Evaluation of Beta PIs from the USDA-ARS, NPGS for Rhizoctonia root and crown rot resistance, 2017. Plant Dis. Manag. Rep. 12:CF205.

Frese, L., B. Desprez, and D. Ziegler. 2001. Potential of genetic resources and breeding strategies for base-broadening in Beta, p. 295-309. In: H.D. Cooper, C. Spillane, and T. Hodgkin (eds.). Broadening the genetic base of crop production. FAO, IBPRGI jointly with CABI, Rome.

Gaskill, J.O. 1968. Breeding for Rhizoctonia resistance in sugarbeet. J. Amer. Soc. Sugar Beet Technol. 15:107-119.

Gaskill, J.O., D.L. Mumford, and E.G. Ruppel. 1970. Preliminary report on breeding sugarbeet for combined resistance to leaf spot, curly top, and Rhizoctonia. J. Amer. Soc. Sugar Beet Technol. 16:207-213.
Gaskill, J.O., C.L. Schneider, A.M. Murphy, and G.E. Coe. 1967. Breeding for combined resistance to leaf spot and curly top in sugar beet. J. Amer. Soc. Sugar Beet Technol. 14:518-537. $<$ https://bsdf-assbt.org/>.

Germplasm Resources Information Network. 2020. U.S. Department of Agriculture, Agricultural Research Service. Beltsville, MD. 12 Sept. 2018. <http://www.ars-grin.gov/>.

Godfrey, L.D. and K.V. Yeargan. 1987. Effects and interactions of early season pests on alfalfa yield in Kentucky. J. Econ. Entomol. 80:248256.

Goldman, I.L. 1996. A list of germplasm releases from the University of Wisconsin Table Beet Breeding Program, 1964-1992. HortScience 31:880-881

Gugino, B.K., J.W. Ludwig, and G.S. Abawi. 2008. Evaluation of fungicides for the control of pocket rot and leaf spot of table beet, 2007. Plant Dis. Mgt. Rpt. 2:V156. (online). APS Press, St. Paul, MN. 10 May 2020. <https://www. plantmanagementnetwork.org.ezproxy.library.wisc. edu/pub/trial/PDMR/reports/2008/V156.pdfs.

Halloin, J.M., J.W. Saunders, J.C. Theurer, and J.M. McGrath. 2000. Registration of EL51 sugarbeet germplasm with resistance to Rhizoctonia crown and root rot. Crop Sci. 40:586587.

Hanson, L. 2010. Interaction of Rhizoctonia solani and Rhizopus stolonifer causing root rot of sugar beet. Plant Dis. 94:504-509.

Hanson, L.E. and L. Panella. 2007. Rhizoctonia root rot resistance of Beta PIs from the USDAARS NPGS, 2006. Plant Dis. Mgt. Rpt. 1:V023. (online). APS Press, St. Paul, MN. 10 May 2020. $<$ http://www.plantmanagementnetwork.org. ezproxy.library.wisc.edu/pub/trial/PDMR/reports/ 2007/V023.pdf $>$.

Harveson, R.M. 2003. Common weeds serving as alternate hosts for pathogens of dry-edible beans and sugar beets in the Nebraska Panhandle. Phytopathology 93:S34, doi: 10.1094/ PHYTO.2003.93.6.S1.

Harveson, R.M., G.L. Hein, J.A. Smith, R.G. Wilson, and C.D. Yonts. 2002. An integrated approach to cultivar evaluation and selection for improving sugar beet profitability: A successful case study for the Central High Plains. Plant Dis. 86:192-204.

Harveson, R.M. and C.M. Rush. 1994. Evaluation of fumigation and Rhizomania-tolerant cultivars for control of a root rot disease complex of sugar beets. Plant Dis. 78:1197-1202.

Hatcher, P.E. 1995. Three-way interactions between plant pathogenic fungi, herbivorous insects and their host plants. Biol. Rev. Camb. Philos. Soc. 70:639-694.

Hecker, R.J. and J.O. Gaskill. 1972. Registration of FC 701 and FC 702 sugarbeet germplasm. Crop Sci. 12:400

Hecker, R.J. and E.G. Ruppel. 1975. Inheritance of resistance to Rhizoctonia root rot in sugarbeet. Crop Sci. 15:487-490.

Hecker, R.J. and E.G. Ruppel. 1976. Polyploid and maternal effects on Rhizoctonia root rot resistance in sugarbeet. Euphytica 25:419-423.

Hecker, R.J. and E.G. Ruppel. 1977a. Registration of diploid and tetraploid FC 701/4 and FC 703 sugarbeet germplasm. Crop Sci. 17:678.

Hecker, R.J. and E.G. Ruppel. 1977b. Rhizoctonia root-rot resistance in sugarbeet: Breeding and related research. J. Amer. Soc. Sugar Beet Technol. 19:246-256

Hecker, R.J. and E.G. Ruppel. 1985. Registration of Rhizoctonia root rot resistant sugarbeet germplasm FC701/6, FC702/7, and FC705/1. Crop Sci. 25:374. 
Hecker, R.J. and E.G. Ruppel. 1988. Registration of Rhizoctonia root rot resistant sugarbeet germplasm FC709. Crop Sci. 28:1039-1040.

Hecker, R.J. and E.G. Ruppel. 1991. Registration of Rhizoctonia root rot resistant sugarbeet germplasm FC710. Crop Sci. 31:494.

Herr, L.J. 1970. Resistant sugar beets show promise in Ohio. Ohio Rep. Res. Dev. 55:50-51.

Jacobsen, B.J. 1997. Role of plant pathology in integrated pest management. Annu. Rev. Phytopathol. 35:373-391.

Jacobsen, B.J. 2006. Root rot diseases of sugar beet. Proc. Natl. Acad. Sci. USA 110:9-19.

Jacobsen, B., K. Kephart, N. Zidack, M. Johnston, and J. Ansley. 2004. Effect of fungicide and fungicide application timing on reducing yield loss to Rhizoctonia Crown and Root Rot. Sugarbeet Res. Ext. Rpt. 35:224-226.

Jiang, J., L. Ding, T.J. Michailides, H. Li, and Z. Ma. 2009. Molecular characterization of field azoxystrobin-resistant isolates of Botrytis cinerea. Pestic. Biochem. Physiol. 93:72-76.

Kataria, H.R. and U. Gisi. 1996. Chemical control of Rhizoctonia species, p. 537-547. In: B. Sneh, S. Jabaji-Hare, S. Neate, and G. Dijst (eds.). Rhizoctonia species: Taxonomy, molecular biology, ecology, pathology, and disease control. Kluwer Academic Publishers, Dordrecht, The Netherlands.

Khan, M., D. Franzen, M. Boetel, A. Chanda, A. Sims, and T. Peters. 2020. 2020 Sugarbeet production guide A1698. N. Dak. State Univ. Ext. and Univ. of Minn. Ext. 20 May 2020. $<$ https://www.ag.ndsu.edu/publications/crops/ sugarbeet-production-guide/a1698.pdf>.

Kiewnick, S., B.J. Jacobsen, A. Braun-Kiewnick, J.L.A. Eckhoff, and J.W. Bergman. 2001. Integrated control of Rhizoctonia crown and root rot of sugar beet with fungicides and antagonistic bacteria. Plant Dis. 85:718-722.

Kirk, W.W., P.S. Wharton, R.L. Schafer, P. Tumbalam, S. Poindexter, C. Guza, R. Fogg, T. Schlatter, J. Stewart, L. Hubbell, and D. Ruppal. 2008. Optimizing fungicide timing for the control of Rhizoctonia crown and root rot of sugar beet using soil temperature and plant growth stages. Plant Dis. 92:10911098.

Kuznetsova, A., P.B. Brockhoff, and R.H.B. Christensen. 2017. ImerTest package: Tests in linear mixed effects models. J. Stat. Softw. 82:1-26.

Liddell, T.M. and J.K. Kruschke. 2018. Analyzing ordinal data with metric models: What could possibly go wrong? J. Expt. Soc. Psychol. 79:328-348

Liu, Y., A. Qi, and M.F.R. Khan. 2019. Agedependent resistance to Rhizoctonia solani in sugar beet. Plant Dis. 103:2322-2329.

Lunos, A.G. 2016. Geographic distribution and severity of strobilurin fungicide resistance among Rhizoctonia solani on rice in southwestern Louisiana. La. State Univ. Agr. Mech. College, Baton Rouge, Master's Thesis. $<$ https://digitalcommons.1su.edu/gradschool_ theses/3064/>.

Ma, Z., D. Felts, and T.J. Michailides. 2003. Resistance to azoxystrobin in Alternia isolates from pistachio in California. Pestic. Biochem. Physiol. 77:66-74.

Madden, V.M., G. Hughes, and F. van den Bosch. 2007. The study of plant disease epidemics. APS Press, St. Paul, MN.

McGrath, J.M. and L. Panella. 2019. Sugar beet breeding, p. 167-218. In: I. Goldman (ed.). Plant Breeding Rev., 42. Wiley, Hoboken, NJ.

McKeen, W.E. 1949. A study of sugar beet rootrot in Southern Ontario. Can. J. Res. 27c(6):284311.
Nagendran, S., R. Hammerschmidt, and J.M. McGrath. 2009. Identification of sugar beet germplasm EL51 as a source of resistance to post-emergence Rhizoctonia damping-off. Eur. J. Plant Pathol. 123:461-471.

Naito, S., T. Yamaguchi, and T. Sugimoto. 1978 Anastomosis groups of Rhizoctonia solani Kühn isolated from blighted leaves of sugar beets. Res. Bul. Hokkaido Natl. Agr. Expt. Sta. 121:71-77.

Naito, S., T. Yamaguchi, T. Sugimoto, and I. Fujisawa. 1976. Studies on root rot of sugar beets, 6: Anastomosis groups of Rhizoctonia solani Kühn isolated from diseased petiols, crowns and roots. Hokkaido Natl. Agr. Expt. Sta. 17:37-44.

Naito, S., T. Yamaguchi, T. Sugimoto, and Y. Homma. 1993. A simple method for the longtime culture storage of Rhizoctonia spp. on barleys' grains. Annu. Rpt. Plant Protection North Japan 44:20-23.

Natti, J.J. 1953. Dry rot of table beets. N. Y. State Farm Res. (July): 7

Nowakowska, M., M. Nowicki, U. Kłosin'ska, R. Maciorowski, and E.U. Kozik. 2014. Appraisal of artificial screening techniques of tomato to accurately reflect field performance of the late blight resistance. PLoS One 9:e109328.

Ogoshi, A. 1985. Anastomosis and intraspecific groups of Rhizoctonia solani and binucleate Rhizoctonia. Fitopatol. Bras. 10:372-390.

Ogoshi, A. 1987. Ecology and pathogenicity of anastomosis and intraspecific groups of Rhizoctonia solani Kühn. Annu. Rev. Phytopathol. $25: 125-143$

Ogoshi, A. 1996. Introduction: The genus Rhizoctonia, p. 1-9. In: B. Sneh, S. Jabaji-Hare, S. Neate, and G. Dijst (eds.). Rhizoctonia species: Taxonomy, molecular biology, ecology, pathology, and disease control. Kluwer Academic Publishers, Dordrecht, The Netherlands.

Ohkura, M., G.S. Abawi, C.D. Smart, and K.T. Hodge. 2009. Diversity and aggressiveness of Rhizoctonia solani and Rhizoctonia-like fungi on vegetables in New York. Plant Dis. 93:615624.

Olaya, G., G.S. Abawi, and J. Barnard. 1994. Response of Rhizoctonia solani and binucleate Rhizoctonia to five fungicides and control of pocket rot of table beets with foliar sprays. Plant Dis. 78:1033-1037.

Olaya, G., C. Buitrago, D. Pearsaul, H. Sierotzki, and A. Tally. 2012. Detection of resistance to QoI fungicides in Rhizoctonia solani isolates from rice. Phytopathology 102:S4.88.

Panella, L. 1999. Registration of FC709-2 and FC727 sugarbeet germplasms resistant to Rhizoctonia root rot and Cercospora leaf spot. Crop Sci. 39:298-299.

Panella, L. 2000. Evaluation of Rhizoctonia-rootrot-resistant germplasm released by the USDAARS Sugar Beet Research Unit over 30 years, 1999. Biological and Cultural Test for Control of Plant Diseases. APS Press, St. Paul, MN. 15:36.

Panella, L., L.G. Campbell, I.A. Eujayl, R.T. Lewellen, and J.M. McGrath. 2015a. USDAARS sugarbeet releases and breeding over the past 20 years. J. Sugar Beet Res. 52:40-85.

Panella, L., A.L. Fenwick, A.L. Hill, M. McClintock, and T. Vagher. 2008. Rhizoctonia root rot resistance of Beta PIs from the USDA-ARS NPGS, 2007. Plant Dis. Mgt. Rpt. (online) 2:V057. APS Press, St. Paul, MN.

Panella, L. and L.E. Hanson. 2001. Publicly released sugar beet germplasm evaluated for resistance to Rhizoctonia root rot, 2000. Biol. Cult. Tests Control Plant Dis. 16:F18.
Panella, L. and R.T. Lewellen. 2007. Broadening the genetic base of sugar beet: Introgression from wild relatives. Euphytica 154:383-400.

Panella, L. and E.G. Ruppel. 1998. Screening of Beta PIs from the USDA-ARS National Plant Germplasm System for resistance to Rhizoctonia root rot, 1997. Biol. Cult. Test Control Plant Dis. 13:151

Panella, L., T. Vagher, and A.L. Fenwick. 2015b. USDA-ARS germplasms evaluated for resistance to Rhizoctonia crown and root rot in Ft. Collins, CO, 2014. Plant Dis. Manag. Rep. 9:FC138.

Parmeter, J.R., Jr. 1970. Rhizoctonia solani, biology and pathology. Univ. Calif. Press, Los Angeles, CA.

Pethybridge, S.J., J.R. Kikkert, L.E. Hanson, and S.C. Nelson. 2018. Challenges and prospects for building resilient disease management strategies and tactics for the New York table beet industry. Agronomy 8:112.

R Core Team. 2018. R: A language and environment for statistical computing. R Foundation for Statistical Computing, Vienna, Austria. $<$ https://www.R-project.org/>.

Ruppel, E.G. 1972. Correlation of cultural characters and source of isolates with pathogenicity of Rhizoctonia solani from sugar beet. Phytopathology 62:202-205.

Ruppel, E.G., C.L. Schneider, R.J. Hecker, and G.J. Hogaboam. 1979. Creating epiphytotics of Rhizoctonia root rot and evaluating for resistance to Rhizoctonia solani in sugarbeet field plots. Plant Dis. Rep. 63:518-522.

Rush, C.M., D.E. Carling, R.M. Harveson, and J.T. Mathieson. 1994. Prevalence and pathogenicity of anastomosis groups of Rhizoctonia solani from wheat and sugar beet in Texas. Plant Dis. 78:349-352.

Schandry, N. 2017. A practical guide to visualization and statistical analysis of $R$. solanacearum infection data using R. Front. Plant Sci. 8:623.

Schuster, M.L. and L. Harris. 1960. Incidence of Rhizoctonia crown rot of sugar beets in irrigated crop rotation. J. Amer. Soc. Sugar Beet Technol. 11:128-136.

Schneider, C.L., E.G. Ruppel, R.J. Hecker, and G.J. Hogaboam. 1982. Effect of soil deposition in crowns on development of Rhizoctonia root rot in sugar beet. Plant Dis. 66:408-410.

Scholten, O.E., L.W. Panella, T.S.M. De Bock, and W. Lange. 2001. A greenhouse test for screening sugar beet (Beta vulgaris) for resistance to Rhizoctonia solani. Eur. J. Plant Pathol. 107:161-166.

Sneh, B., S. Jabaji-Hare, S. Neate, and G. Dijst (eds.). 1996. Rhizoctonia species: Taxonomy, molecular biology, ecology, pathology, and disease control. Kluwer Academic Publishers, Dordrecht, The Netherlands.

Stevenson, K.L., D.B. Langston, Jr., and K.W. Seebold. 2004. Resistance to azoxystrobin in the gummy stem blight pathogen documented in Georgia. Online. Plant Health Prog. 16 June 2020. <https://apsjournals.apsnet.org/doi/pdf/ 10.1094/PHP-2004-1207-01-RS >.

Stivers, L. n.d. Crop Profile: Beets in New York. Cornell Coop. Ext. Bul. 27 June 2019. <http://pmep.cce. cornell.edu/fqpa/crop-profiles/beet.html>.

Strausbaugh, C.A., I.A. Eujayl, and P. Foote. 2013b. Selection for resistance to the Rhizoctonia-bacterial root rot complex in sugar beet. Plant Dis. 97:93-100.

Strausbaugh, C.A., I.A. Eujayl, and L.W. Panella. 2013a. Interaction of sugar beet host resistance and Rhizoctonia solani AG-2-2 IIIB strains. Plant Dis. 97:1175-1180. 
Strausbaugh, C.A., I.A. Eujayl, L.W. Panella, and L.E. Hanson. 2009. Genetic diversity and pathogenicity of Rhizoctonia on sugarbeet. Proc. Conf. Amer. Soc. Sugar Beet Technol.

Strausbaugh, C.A., I.A. Eujayl, L.W. Panella, and L.E. Hanson. 2011. Virulence, distribution and diversity of Rhizoctonia solani from sugar beet in Idaho and Oregon. Can. J. Plant Pathol. 33:210-226.

Strausbaugh, C.A. and A.M. Gillen. 2009. Sugar beet root rot at harvest in the US Intermountain West. Can. J. Plant Pathol. 31:232-240.

Stump, W.L., G.D. Franc, R.M. Harveson, and R.G Wilson. 2004. Strobilurin fungicide timing for Rhizoctonia Root and Crown Rot suppression in sugarbeet. J. Sugar Beet Res. 41:17-37.

Stump, W.L., G.D. Franc, S.D. Miller, and R.G. Wilson. 2002. Azoxystrobin and post emergence herbicide combinations for Rhizoctonia and weed management in sugarbeet. J. Sugar Beet Res. 39:37-58.

Sumner, D.R. and D.K. Bell. 1982. Root diseases induced in corn by Rhizoctonia solani and Rhizoctonia zeae. Phytopathology 72:86-91.

Sumner, D.R. and D.K. Bell. 1994. Survival of Rhizoctonia spp. and root diseases in a rotation of corn, snap bean, and peanuts in microplots. Phytopathology 84:113-118.

U.S. Department of Agriculture. 2017. National Agricultural Statistics Service, Quick Stats. 11 Oct. 2019. <https://quickstats.nass.usda.gov>.

Vaghefi, N., F.S. Hay, J.R. Kikkert, and S.J. Pethybridge. 2016. Genotypic diversity and resistance to azoxystrobin of Cercospora beticola on processing table beet in New York. Plant Dis. 100:1466-1473.

Venables, W.N. and B.D. Ripley. 2002. Modern applied statistics with S. 4th ed. Springer, New York, NY

Vincelli, P. and E. Dixon. 2002. Resistance to QOI (Strobilurin-like) fungicides in isolates of Pyricularia grisea from perennial ryegrass. Plant Dis. 86:235-240.

Wang, M. and I.L. Goldman. 1999. Genetic distance and diversity in table beet and sugar beet accessions measured by randomly amplified polymorphic DNA. J. Amer. Soc. Hort. Sci. 124:630-635.

Watanabe, B. and A. Matsuda. 1966. Studies on the grouping of Rhizoctonia solani Kuehn pathogenic to upland crops. Appointment Expt. (Plant Dis. Insect Pests) No. 7. Agr. For. Fisheries Res. Council Ibaraki Agr. Expt. Sta. 7:1-131.

Weiland, J.J., G.A. Smith, and L. Panella. 1999. Greenhouse assay for the evaluation of sugarbeet resistance to Rhizoctonia root rot. J. Sugar Beet Res. 30:211-212.

Windels, C.E. and J.R. Brantner. 2002. Timing applications of Quadris for control of Rhizoctonia on sugarbeet. Sugarbeet Res. Ext. Rpt. 33:182-195

Windels, C.E. and J.R. Brantner. 2005. Earlyseason application of azoxystrobin to sugarbeet for control of Rhizoctonia solani AG 4 and AG 2-2. J. Sugar Beet Res. 42:1-16.
Windels, C.E., B.J. Jacobsen, and R.M. Harveson. 2009. Rhizoctonia root and crown rot, p. 33-36. In: R.M. Harveson, L.E. Hanson, and G.L. Hein (eds.). Compendium of beet diseases and pests. 2nd ed. APS Press, St. Paul, MN.

Windels, C.E. and D.J. Nabben. 1989. Characterization and pathogenicity of anastomosis groups of Rhizoctonia solani isolated from Beta vulgaris. Phytopathology 79:83-88.

Windels, C.E., L. Panella, and E.G. Ruppel. 1995. Sugarbeet germplasm resistant to Rhizoctonia root and crown rot withstands disease caused by several pathogenic isolates of Rhizoctonia solani AG-2-2. Sugarbeet Res. Ext. Rpt. 26:179-185.

Wisconsin Alumni Research Foundation. 2019. WARF: P110151US01. Colorful New Table Beets. 10 July 2019. <https://www.warf.org/ technologies/agriculture/plant-varieties/summary/ colorful-new-table-beets-p110151us01.cmsx >.

Woodward, S. and R.B. Pearce. 1988. Responses of Sitka spruce callus to challenge with wood decay fungi. Eur. J. Forest Pathol. 18:217229.

Yamaguchi, T., T. Sugimoto, and S. Naito. 1977. Studies on root rot of sugar beets, 7: Further studies of comparison with sugar beet varieties and strains for resistance to Rhizoctonia root rot. Hokkaido Natl. Agr. Expt. Sta. 18:131141. 


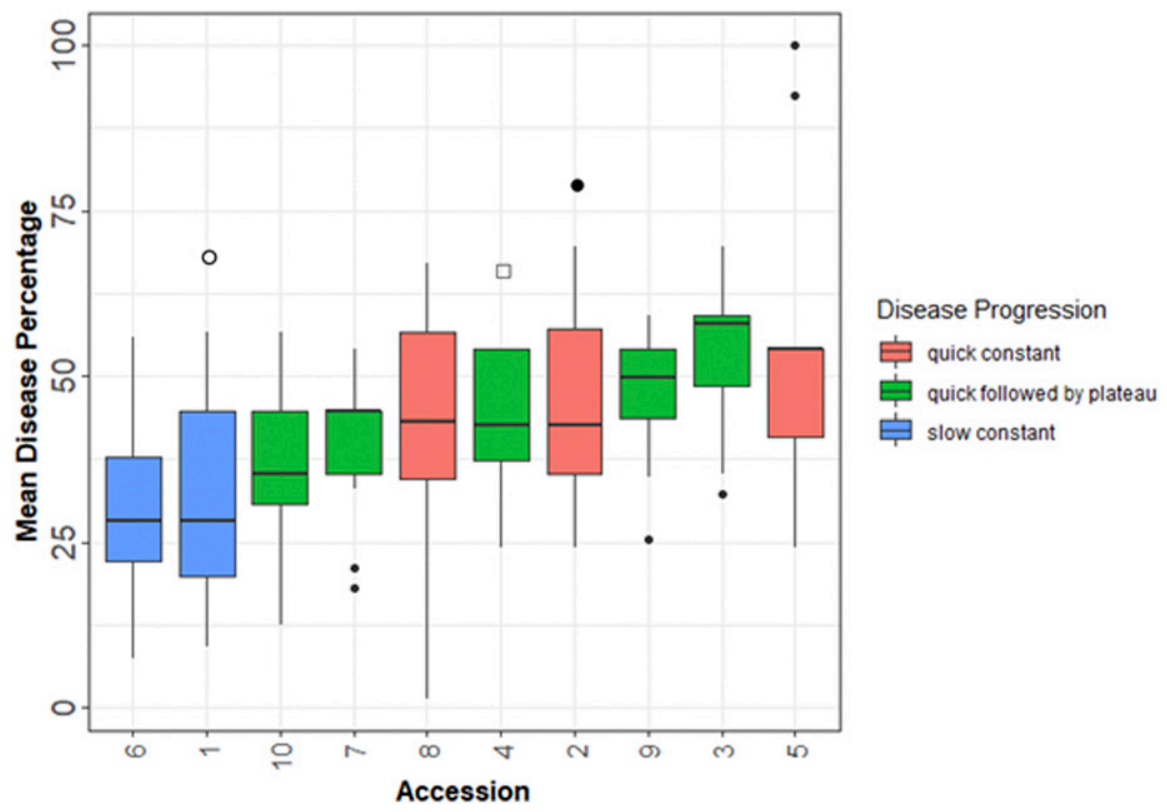

Supplemental Fig. 1. The response of 10 Beta vulgaris subsp. vulgaris commercial cultivars and publicly available accessions to inoculation with Rhizoctonia solani AG 2-2 isolate R1 in a greenhouse screen. Accession codes are in Table 3. Slopes between zero days postinoculation and the first evaluation period and the first evaluation period and second evaluation period were determined from spline regression and used to categorize accessions according to their disease progression behavior. Disease progression categories included the following: slow followed by spike (Slope $1 \leq 1.25$, Slope $2>$

1.25 ), quick followed by plateau (Slope $1>1.25$, Slope $2 \leq 1.25$ ), quick constant (Slope $1>1.25$, Slope $2>1.25$ ), and slow constant (Slope $1 \leq 1.25$, Slope $2 \leq 1.25$ ). ${ }^{\circ}$ FC709-2, resistant sugar beet control; ${ }^{\bullet}$ FC901/C817, susceptible sugar beet control; ${ }^{\square}$ W364B, table beet inbred control.

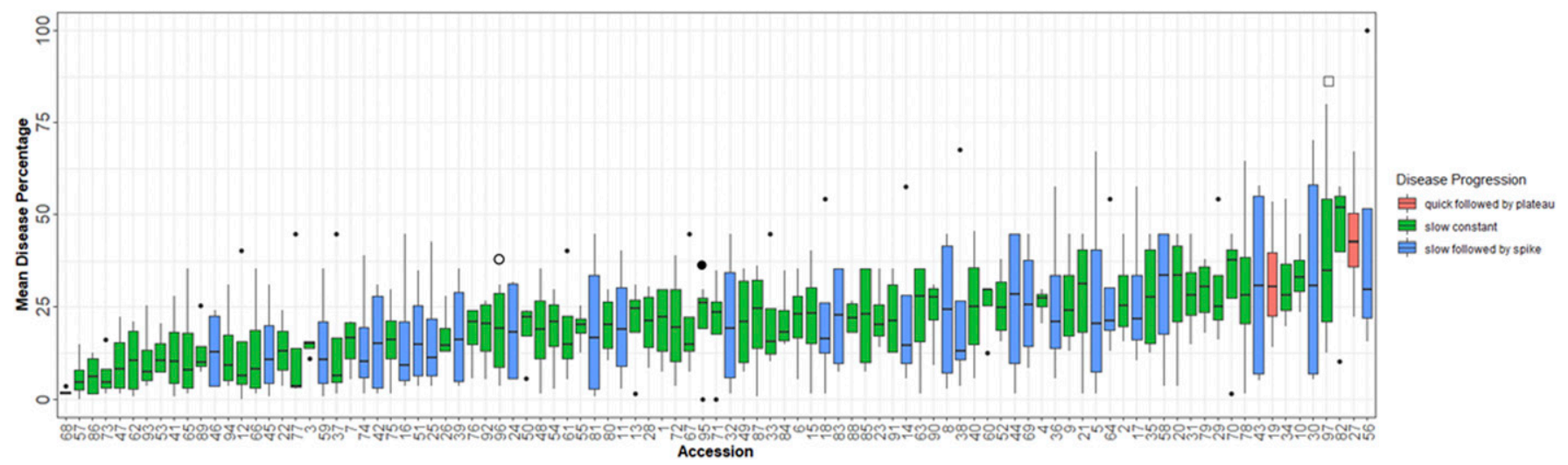

Supplemental Fig. 2. The response of 97 Beta vulgaris subsp. vulgaris PI accessions to inoculation with Rhizoctonia solani AG 2-2 isolate R1 in a greenhouse screen. Accession codes are in Table 1. Slopes between zero days postinoculation and the first evaluation period and the first evaluation period and second evaluation period were determined from spline regression and used to categorize accessions according to their disease progression behavior. Disease progression categories included the following: slow followed by spike (Slope $1 \leq 1.25$, Slope $2>1.25$ ), quick followed by plateau (Slope $1>1.25$, Slope $2 \leq$ 1.25), quick constant (Slope $1>1.25$, Slope $2>1.25$ ), and slow constant (Slope $1 \leq 1.25$, Slope $2 \leq 1.25$ ). ${ }^{\circ}$ FC709-2, resistant sugar beet control; $\bullet^{\bullet}$ FC901/ C817, susceptible sugar beet control; ${ }^{\square}$ W364B, table beet inbred control. 


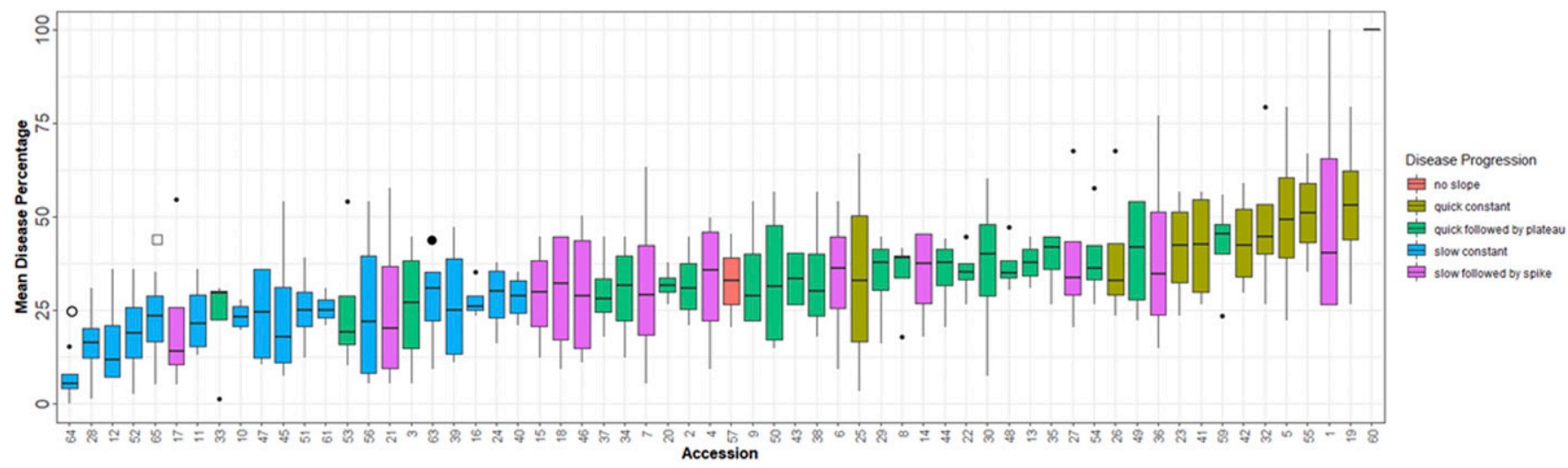

Supplemental Fig. 3. The response of 62 Beta vulgaris subsp. vulgaris commercial cultivars and publicly available inbred accessions to inoculation with Rhizoctonia solani AG 2-2 isolate R1 in a greenhouse screen. Accession codes are in Table 2. Slopes between zero days postinoculation and the first evaluation period and the first evaluation period and second evaluation period were determined from spline regression and used to categorize accessions according to their disease progression behavior. Disease progression categories included the following: slow followed by spike (Slope $1 \leq 1.25$, Slope $2>1.25$ ), quick followed by plateau (Slope $1>1.25$, Slope $2 \leq 1.25$ ), quick constant (Slope $1>1.25$, Slope $2>1.25$ ), and slow constant (Slope $1 \leq 1.25$, Slope $2 \leq 1.25$ ). ${ }^{\circ}$ FC709-2, resistant sugar beet control; $\bullet^{\bullet} \mathrm{FC} 901 / \mathrm{C} 817$, susceptible sugar beet control; ${ }^{\square} \mathrm{W} 364 \mathrm{~B}$, table beet inbred control.

Supplemental Table 1. The slopes predicted by spline regression for 10 Beta vulgaris subsp. vulgaris commercial cultivars and publicly available accessions to inoculation with Rhizoctonia solani AG 2-2 isolate R1. Accession codes are in Table 3. Slope 1 was calculated for the diseased tissue percentage between 0 and 3 weeks postinoculation. Slope 2 was calculated between 3 and 5 weeks postinoculation. Slopes were then used to assign each accession to a disease progression categories according to the following: slow followed by spike (Slope $1 \leq 1.25$, Slope $2>1.25$ ), quick followed by plateau (Slope 1 $>1.25$, Slope $2 \leq 1.25$ ), quick constant (Slope $1>1.25$, Slope $2>1.25$ ), and slow constant (Slope $1 \leq$ 1.25 , Slope $2 \leq 1.25$ ).

\begin{tabular}{lrr}
\hline Accession code & Slope 1 (SE) & Slope 2 (SE) \\
\hline $1^{\mathrm{z}}$ & $1.23(0.47)$ & $0.91(1.35)$ \\
$2^{\mathrm{y}}$ & $1.69(0.47)$ & $1.61(1.35)$ \\
3 & $2.30(0.47)$ & $0.61(1.34)$ \\
$4^{\mathrm{x}}$ & $2.06(0.47)$ & $-0.33(1.35)$ \\
5 & $2.21(0.47)$ & $1.57(1.55)$ \\
6 & $1.04(0.47)$ & $1.23(0.56)$ \\
7 & $1.52(0.47)$ & $1.09(1.35)$ \\
8 & $1.52(0.47)$ & $1.64(1.35)$ \\
9 & $2.06(0.47)$ & $0.47(1.35)$ \\
10 & $1.57(0.47)$ & $0.51(1.35)$ \\
\hline
\end{tabular}

${ }^{\mathrm{z}} \mathrm{FC} 709-2$, resistant sugar beet control.

${ }^{\mathrm{y}} \mathrm{FC} 901 / \mathrm{C} 817$, susceptible sugar beet control.

${ }^{\mathrm{x}} \mathrm{W} 364 \mathrm{~B}$, table beet inbred control. 
Supplemental Table 2. The slopes predicted by spline regression for 97 Beta vulgaris subsp. vulgaris PI accessions to inoculation with Rhizoctonia solani AG 2-2 isolate R1. Accession codes are in Table 1. Slope 1 was calculated for the diseased tissue percentage between 0 and 3 weeks postinoculation. Slope 2 was calculated between 3 and 5 weeks postinoculation. Slopes were then used to assign each accession to a disease progression categories according to the following: slow followed by spike (Slope $1 \leq 1.25$, Slope $2>1.25$ ), quick followed by plateau (Slope $1>1.25$, Slope $2 \leq 1.25$ ), quick constant (Slope $1>1.25$, Slope $2>1.25$ ), and slow constant (Slope $1 \leq 1.25$, Slope $2 \leq 1.25$ ).

\begin{tabular}{|c|c|c|}
\hline Accession code & Slope 1 (SE) & Slope $2(\mathrm{SE})$ \\
\hline 1 & $0.47(0.59)$ & $1.06(0.95)$ \\
\hline 2 & $1.25(0.58)$ & $-0.90(2.34)$ \\
\hline 3 & $0.51(0.57)$ & $0.06(2.33)$ \\
\hline 4 & $1.06(0.57)$ & $-0.33(2.26)$ \\
\hline 5 & $0.22(0.59)$ & $3.08(2.36)$ \\
\hline 6 & $0.51(0.56)$ & $1.22(2.29)$ \\
\hline 7 & $0.51(0.57)$ & $0.19(2.31)$ \\
\hline 8 & $0.31(0.58)$ & $2.39(2.32)$ \\
\hline 9 & $0.65(0.59)$ & $1.11(2.26)$ \\
\hline 10 & $1.11(0.57)$ & $0.40(2.22)$ \\
\hline 11 & $0.27(0.57)$ & $1.85(2.31)$ \\
\hline 12 & $0.23(0.57)$ & $0.95(2.26)$ \\
\hline 13 & $0.67(0.59)$ & $0.42(2.38)$ \\
\hline 14 & $0.32(0.57)$ & $2.09(2.31)$ \\
\hline 15 & $0.51(0.57)$ & $1.28(2.30)$ \\
\hline 16 & $0.18(0.56)$ & $1.75(2.29)$ \\
\hline 17 & $0.46(0.57)$ & $2.29(2.30)$ \\
\hline 18 & $0.24(0.56)$ & $2.33(2.29)$ \\
\hline 19 & $1.31(0.57)$ & $-0.50(2.42)$ \\
\hline 20 & $1.15(0.57)$ & $-0.38(2.38)$ \\
\hline 21 & $0.83(0.57)$ & $0.79(2.31)$ \\
\hline 22 & $0.27(0.59)$ & $0.94(2.38)$ \\
\hline 23 & $0.70(0.57)$ & $0.50(2.33)$ \\
\hline 24 & $0.21(0.57)$ & $1.51(2.22)$ \\
\hline 25 & $0.15(0.57)$ & $2.20(2.39)$ \\
\hline 26 & $0.58(0.57)$ & $0.22(2.26)$ \\
\hline 27 & $1.78(0.57)$ & $-0.62(2.38)$ \\
\hline 28 & $0.47(0.57)$ & $1.14(2.33)$ \\
\hline 29 & $0.88(0.57)$ & $0.78(2.26)$ \\
\hline 30 & $0.27(0.59)$ & $3.92(2.36)$ \\
\hline 31 & $1.17(0.59)$ & $-0.22(2.36)$ \\
\hline 32 & $0.18(0.59)$ & $1.94(2.26)$ \\
\hline 33 & $0.70(0.59)$ & $0.46(2.31)$ \\
\hline 34 & $1.06(0.59)$ & $0.60(2.26)$ \\
\hline 35 & $0.54(0.57)$ & $1.73(2.26)$ \\
\hline 36 & $0.45(0.58)$ & $1.71(2.23)$ \\
\hline 37 & $0.28(0.59)$ & $0.99(2.30)$ \\
\hline 38 & $0.13(0.58)$ & $3.05(2.32)$ \\
\hline 39 & $0.20(0.57)$ & $1.61(2.26)$ \\
\hline 40 & $0.49(0.59)$ & $1.48(2.26)$ \\
\hline 41 & $0.14(0.59)$ & $1.28(2.38)$ \\
\hline 42 & $0.11(0.57)$ & $1.64(2.27)$ \\
\hline 43 & $0.27(0.57)$ & $3.08(2.26)$ \\
\hline 44 & $0.30(0.59)$ & $2.32(2.30)$ \\
\hline 45 & $0.10(0.59)$ & $1.43(2.31)$ \\
\hline 46 & $0.13(0.57)$ & $1.40(2.31)$ \\
\hline 47 & $0.10(0.59)$ & $0.87(2.26)$ \\
\hline 48 & $0.47(0.57)$ & $0.80(2.27)$ \\
\hline 49 & $0.38(0.59)$ & $1.45(2.30)$ \\
\hline 50 & $0.54(0.57)$ & $0.47(2.26)$ \\
\hline 51 & $0.23(0.59)$ & $1.59(2.36)$ \\
\hline 52 & $0.94(0.57)$ & 0.09 (2.27) \\
\hline 53 & $0.34(0.59)$ & $0.46(2.31)$ \\
\hline 54 & $0.38(0.57)$ & $1.05(2.27)$ \\
\hline 55 & $0.71(0.59)$ & $0.18(2.43)$ \\
\hline 56 & $0.83(0.59)$ & $3.17(2.36)$ \\
\hline 57 & $0.14(0.59)$ & $0.30(2.31)$ \\
\hline 58 & $0.56(0.57)$ & $2.36(2.39)$ \\
\hline 59 & $0.11(0.58)$ & $1.63(2.32)$ \\
\hline 60 & $0.88(0.59)$ & $0.38(2.38)$ \\
\hline 61 & $0.33(0.59)$ & $1.30(2.30)$ \\
\hline 62 & $0.09(0.59)$ & $1.10(2.30)$ \\
\hline 63 & $0.75(0.58)$ & $0.40(2.23)$ \\
\hline 64 & $0.55(0.57)$ & $2.08(2.38)$ \\
\hline 65 & $0.10(0.59)$ & $1.24(2.26)$ \\
\hline 66 & $0.20(0.57)$ & $1.35(2.39)$ \\
\hline 67 & $0.42(0.58)$ & $1.06(2.30)$ \\
\hline 68 & $0.06(0.56)$ & $0.02(2.29)$ \\
\hline
\end{tabular}

(Continued on next page) 
Supplemental Table 2. (Continued) The slopes predicted by spline regression for 97 Beta vulgaris subsp. vulgaris $\mathrm{PI}$ accessions to inoculation with Rhizoctonia solani AG 2-2 isolate R1. Accession codes are in Table 1. Slope 1 was calculated for the diseased tissue percentage between 0 and 3 weeks

postinoculation. Slope 2 was calculated between 3 and 5 weeks postinoculation. Slopes were then used to assign each accession to a disease progression categories according to the following: slow followed by spike (Slope $1 \leq 1.25$, Slope $2>1.25$ ), quick followed by plateau (Slope $1>1.25$, Slope $2 \leq 1.25$ ), quick constant (Slope $1>1.25$, Slope $2>1.25$ ), and slow constant (Slope $1 \leq 1.25$, Slope $2 \leq 1.25$ ).

\begin{tabular}{llr}
\hline Accession code & Slope 1 (SE) & Slope 2 (SE) \\
\hline 69 & $0.50(0.59)$ & $2.29(2.47)$ \\
70 & $0.77(0.57)$ & $1.47(2.31)$ \\
71 & $0.49(0.58)$ & $1.12(2.32)$ \\
72 & $0.55(0.57)$ & $1.05(2.42)$ \\
73 & $0.16(0.59)$ & $0.38(2.38)$ \\
74 & $0.19(0.59)$ & $1.67(2.43)$ \\
75 & $0.30(0.57)$ & $1.04(2.27)$ \\
76 & $0.48(0.57)$ & $0.69(2.27)$ \\
77 & $0.15(0.59)$ & $1.16(2.26)$ \\
78 & $0.69(0.57)$ & $1.67(2.27)$ \\
79 & $0.92(0.59)$ & $0.70(2.31)$ \\
80 & $0.89(0.58)$ & $-0.41(2.23)$ \\
81 & $0.07(0.57)$ & $2.97(2.38)$ \\
82 & $1.20(0.57)$ & $1.30(2.22)$ \\
83 & $0.37(0.59)$ & $1.85(2.38)$ \\
84 & $0.97(0.57)$ & $-0.74(2.38)$ \\
85 & $0.90(0.57)$ & $-0.24(2.33)$ \\
86 & $0.30(0.57)$ & $-0.21(2.28)$ \\
87 & $0.40(0.57)$ & $1.41(2.26)$ \\
88 & $0.71(0.57)$ & $0.53(2.43)$ \\
89 & $0.29(0.57)$ & $0.68(2.26)$ \\
90 & $0.80(0.59)$ & $0.37(2.31)$ \\
91 & $0.52(0.59)$ & $1.28(2.36)$ \\
92 & $0.85(0.57)$ & $-0.47(2.26)$ \\
93 & $0.22(0.58)$ & $0.82(2.39)$ \\
94 & $0.25(0.58)$ & $0.96(2.32)$ \\
$95^{z}$ & $0.54(0.57)$ & $0.80(2.26)$ \\
$96^{y}$ & $0.29(0.57)$ & $1.35(2.27)$ \\
$97^{\mathrm{x}}$ & $1.14(0.48)$ & $1.81(1.87)$ \\
\hline & &
\end{tabular}

${ }^{\mathrm{z}} \mathrm{FC} 901 / \mathrm{C} 817$, susceptible sugar beet control.

${ }^{\mathrm{y}} \mathrm{FC} 709-2$, resistant sugar beet control.

${ }^{\mathrm{x}} \mathrm{W} 364 \mathrm{~B}$, table beet inbred control. 
Supplemental Table 3. The slopes predicted by spline regression for 62 Beta vulgaris subsp. vulgaris commercial cultivars and publicly available inbred accessions to inoculation with Rhizoctonia solani AG 2-2 isolate R1. Accession codes are in Table 2. Slope 1 was calculated for the diseased tissue percentage between 0 and 3 weeks postinoculation. Slope 2 was calculated between 3 and 5 weeks postinoculation. Slopes were then used to assign each accession to disease progression categories according to the following: slow followed by spike (Slope $1 \leq 1.25$, Slope $2>1.25$ ), quick followed by plateau (Slope $1>1.25$, Slope $2 \leq 1.25$ ), quick constant (Slope $1>1.25$, Slope $2>1.25$ ), and slow constant (Slope $1 \leq 1.25$, Slope $2 \leq 1.25$ ).

\begin{tabular}{|c|c|c|}
\hline Accession code & Slope $1(\mathrm{SE})$ & Slope $2(\mathrm{SE})$ \\
\hline 1 & $1.11(0.86)$ & $4.74(1.35)$ \\
\hline 2 & $1.63(0.86)$ & $-1.05(3.26)$ \\
\hline 3 & $1.31(0.86)$ & $-0.72(3.26)$ \\
\hline 4 & $0.82(0.86)$ & $2.50(3.26)$ \\
\hline 5 & $1.58(0.86)$ & $2.33(3.26)$ \\
\hline 6 & $0.96(0.86)$ & $2.19(3.26)$ \\
\hline 7 & $0.87(0.86)$ & $2.17(3.26)$ \\
\hline 8 & $1.30(0.86)$ & $0.81(3.26)$ \\
\hline 9 & $1.37(0.86)$ & $0.36(3.26)$ \\
\hline 10 & $1.18(0.86)$ & $-0.71(3.26)$ \\
\hline 11 & $0.90(0.86)$ & $0.32(3.26)$ \\
\hline 12 & $0.47(0.86)$ & $1.06(3.26)$ \\
\hline 13 & $1.62(0.86)$ & $0.08(3.26)$ \\
\hline 14 & $1.08(0.86)$ & $1.76(3.26)$ \\
\hline 15 & $0.81(0.86)$ & $1.87(3.26)$ \\
\hline 16 & $1.11(0.86)$ & $0.36(3.26)$ \\
\hline 17 & $0.41(0.86)$ & $2.31(3.26)$ \\
\hline 18 & $0.67(0.86)$ & $2.59(3.26)$ \\
\hline 19 & $1.68(0.86)$ & $2.42(3.18)$ \\
\hline 20 & $1.29(0.86)$ & $0.35(3.26)$ \\
\hline 21 & $0.44(0.86)$ & $2.87(3.26)$ \\
\hline 22 & $1.41(0.86)$ & $0.52(3.26)$ \\
\hline 23 & $1.28(0.86)$ & $2.05(3.26)$ \\
\hline 24 & $0.94(0.86)$ & $1.14(3.18)$ \\
\hline 25 & $1.49(0.87)$ & $2.60(3.32)$ \\
\hline 26 & $1.30(0.86)$ & $1.68(3.26)$ \\
\hline 27 & $1.19(0.86)$ & $2.08(3.26)$ \\
\hline 28 & $0.66(0.86)$ & $0.15(3.26)$ \\
\hline 29 & $1.38(0.86)$ & $0.45(3.26)$ \\
\hline 30 & $1.59(0.86)$ & $0.14(3.26)$ \\
\hline 32 & $1.69(0.86)$ & $1.8(3.26)$ \\
\hline 33 & $1.28(0.86)$ & $-1.26(3.26)$ \\
\hline 34 & $1.34(0.86)$ & $-0.09(3.26)$ \\
\hline 35 & $1.61(0.87)$ & $0.28(3.32)$ \\
\hline 36 & $0.85(0.86)$ & $3.72(3.26)$ \\
\hline 37 & $1.63(0.86)$ & $-1.19(3.15)$ \\
\hline 38 & $1.25(0.86)$ & $0.75(3.15)$ \\
\hline 39 & $1.08(0.87)$ & $0.40(3.32)$ \\
\hline 40 & $1.04(0.86)$ & $0.75(3.18)$ \\
\hline 41 & $1.29(0.87)$ & $2.04(3.32)$ \\
\hline 42 & $1.51(0.86)$ & $1.42(3.18)$ \\
\hline 43 & $1.45(0.86)$ & $-0.01(3.18)$ \\
\hline 44 & $1.29(0.86)$ & $0.90(3.18)$ \\
\hline 45 & $0.90(0.86)$ & $0.56(3.15)$ \\
\hline 46 & $0.58(0.86)$ & $2.47(3.15)$ \\
\hline 47 & $1.12(0.87)$ & $-0.30(3.32)$ \\
\hline 48 & $1.44(0.87)$ & $0.57(3.32)$ \\
\hline 49 & $1.69(0.86)$ & $0.04(3.15)$ \\
\hline 50 & $1.43(0.86)$ & $0.11(3.15)$ \\
\hline 51 & $1.17(0.86)$ & $-0.25(3.15)$ \\
\hline 52 & $0.79(0.86)$ & 0.14 \\
\hline 53 & $1.62(0.86)$ & $-1.76(3.15)$ \\
\hline 54 & $2.11(0.86)$ & $-1.58(3.18)$ \\
\hline 55 & $1.47(0.96)$ & $2.26(3.39)$ \\
\hline 56 & $1.10(0.86)$ & $0.12(3.15)$ \\
\hline $57^{z}$ & - & - \\
\hline 59 & $1.58(0.86)$ & $0.94(3.15)$ \\
\hline $60^{y}$ & - & - \\
\hline 61 & $0.98(0.86)$ & $0.42(3.15)$ \\
\hline $63^{x}$ & $1.09(0.87)$ & $0.76(3.32)$ \\
\hline $64^{w}$ & $0.15(0.86)$ & $0.50(3.15)$ \\
\hline $65^{v}$ & $0.98(0.41)$ & $-0.16(3.15)$ \\
\hline
\end{tabular}

${ }^{\mathrm{z}}$ Slopes not provided, observations only at the second evaluation period.

${ }^{\mathrm{y}}$ Slopes not provided, only one observation.

${ }^{\mathrm{x}} \mathrm{FC} 901 / \mathrm{C} 817$, susceptible sugar beet control.

${ }^{\mathrm{w}}$ FC709-2, resistant sugar beet control.

${ }^{\mathrm{v}} \mathrm{W} 364 \mathrm{~B}$, table beet inbred control. 